\title{
The distribution of stars around the Milky Way's central black hole
}

\section{Deep star counts ${ }^{\star}$}

\author{
E. Gallego-Cano ${ }^{1}$, R. Schödel ${ }^{1}$, H. Dong ${ }^{1}$, F. Nogueras-Lara ${ }^{1}$, A. T. Gallego-Calvente ${ }^{1}$, \\ P. Amaro-Seoane ${ }^{2,3,4,5}$, and H. Baumgardt ${ }^{6}$
}

\author{
${ }^{1}$ Instituto de Astrofísica de Andalucía (CSIC), Glorieta de la Astronomía s/n, 18008 Granada, Spain \\ e-mail: lgc@iaa.es \\ 2 Institute of Space Sciences (ICE, CSIC) \& Institut d'Estudis Espacials de Catalunya (IEEC) at Campus UAB, \\ Carrer de Can Magrans s/n, 08193 Barcelona, Spain \\ ${ }^{3}$ Institute of Applied Mathematics, Academy of Mathematics and Systems Science, CAS, Beijing 100190, PR China \\ 4 Kavli Institute for Astronomy and Astrophysics, Beijing 100871, PR China \\ 5 Zentrum für Astronomie und Astrophysik, TU Berlin, Hardenbergstraße 36, 10623 Berlin, Germany \\ ${ }^{6}$ School of Mathematics and Physics, University of Queensland St. Lucia, QLD 4068, Australia
}

Received 18 January 2017 / Accepted 4 October 2017

\begin{abstract}
Context. The existence of dynamically relaxed stellar density cusps in dense clusters around massive black holes is a long-standing prediction of stellar dynamics, but it has so far escaped unambiguous observational confirmation.

Aims. In this paper we aim to revisit the problem of inferring the innermost structure of the Milky Way's nuclear star cluster via star counts, to clarify whether it displays a core or a cusp around the central black hole.

Methods. We used judiciously selected adaptive optics assisted high angular resolution images obtained with the NACO instrument at the ESO VLT. Through image stacking and improved point spread function fitting we pushed the completeness limit about one magnitude deeper than in previous, comparable work. Crowding and extinction corrections were derived and applied to the surface density estimates. Known young, and therefore dynamically not relaxed stars, are excluded from the analysis. Contrary to previous work, we analyse the stellar density in well-defined magnitude ranges in order to be able to constrain stellar masses and ages.

Results. We focus on giant stars, with observed magnitudes $K=12.5-16$, and on stars with observed magnitudes $K \approx 18$, which may have similar mean ages and masses than the former. The giants display a core-like surface density profile within a projected radius $R \leq 0.3 \mathrm{pc}$ of the central black hole, in agreement with previous studies, but their 3D density distribution is not inconsistent with a shallow cusp if we take into account the extent of the entire cluster, beyond the radius of influence of the central black hole. The surface density of the fainter stars can be described well by a single power-law at $R<2 \mathrm{pc}$. The cusp-like profile of the faint stars persists even if we take into account the possible contamination of stars in this brightness range by young pre-main sequence stars. The data are inconsistent with a core-profile for the faint stars. Finally, we show that a 3D Nuker law provides a good description of the cluster structure.

Conclusions. We conclude that the observed density of the faintest stars detectable with reasonable completeness at the Galactic centre, is consistent with the existence of a stellar cusp around the Milky Way's central black hole, Sagittarius A*. This cusp is well developed inside the influence radius of Sagittarius A* and can be described by a single three-dimensional power-law with an exponent $\gamma=1.43 \pm 0.02 \pm 0.1_{\text {sys }}$. This corroborates existing conclusions from Nbody simulations performed in a companion paper. An important caveat is that the faint stars analysed here may be contaminated significantly by dynamically unrelaxed stars that formed about $100 \mathrm{Myr}$ ago. The apparent lack of giants at projected distances of $R \lesssim 0.3 \mathrm{pc}\left(R \lesssim 8^{\prime \prime}\right)$ of the massive black hole may indicate that some mechanism may have altered their distribution or intrinsic luminosity. We roughly estimate the number of possibly missing giants to about 100 .
\end{abstract}

Key words. galaxies: structure - Galaxy: center - Galaxy: structure - infrared: stars

\section{Introduction}

This is the first one of three papers addressing the distribution of stars around Sagittarius A* (Sgr A*), the massive black hole at the centre of the Milky Way. They are closely related, but focus on different methods and stellar populations. In this work we use the method of star counts, while in our other paper (Schödel et al. 2018, referred to as Paper II in the following), we analyse the diffuse light from the unresolved stellar population. Finally, Baumgardt et al. (2018, herafter Paper III) present new $N$-body

\footnotetext{
$\star 19$ additional tables are only available at the CDS via anonymous ftp to cdsarc.u-strasbg. fr (130.79.128.5) or via http://cdsarc.u-strasbg.fr/viz-bin/qcat?J/A+A/609/A26
}

simulations that confirm the agreement between modelling and observations.

The distribution of stars around $\mathrm{Sgr} \mathrm{A}^{*}$ is of great astrophysical interest because it is the only massive black hole where we can test observationally the existence of a stellar cusp. Such a stellar cusp is a prediction of stellar dynamics for the case of a dynamically relaxed cluster and has been derived and studied by analytical, Monte Carlo and $N$-body methods (e.g. Bahcall \& Wolf 1976; Lightman \& Shapiro 1977; Murphy et al. 1991; Baumgardt et al. 2004; Amaro-Seoane et al. 2004; Alexander \& Hopman 2009; Preto \& Amaro-Seoane 2010). These consistent theoretical results have, however, not yet been confirmed observationally. There exist currently only 
measurements of about two dozen systems, where we can actually resolve the radius of influence of the central black hole with several resolution elements (see, e.g. Table 1 in Gültekin et al. 2009). The great distance of most extragalactic systems means that we can only study the light density of hundreds to thousands, or even millions, of stars per resolution element. Since most nuclear star clusters are entities with complex stellar populations, many of which show signs of recent star formation (see review by Böker 2010), measured light densities may frequently be dominated by a small number of bright stars, which are generally too young to be dynamically relaxed. This can lead to ambiguous or erroneous results.

On the other hand, with its distance of only about $8 \mathrm{kpc}$ from Earth, the Galactic centre (GC) allows us to resolve the stars observationally on scales of about 2 milli-parsecs (mpc), assuming diffraction limited observations at about $2 \mu \mathrm{m}$ at an $8-$ $10 \mathrm{~m}$ telescope. At the centre of the Milky Way, a $4 \times 10^{6} M_{\odot}$ (e.g. Boehle et al. 2016; Gillessen et al. 2016) massive black hole, Sagittarius A* (Sgr A*), lies embedded in a $\sim 2.5 \times 10^{7} M_{\odot}$ nuclear star cluster (Schödel et al. 2014a,b; Feldmeier et al. 2014; Chatzopoulos et al. 2015a; Feldmeier-Krause et al. 2017). Therefore, the GC appears to be, in principle, the ideal test case for the existence of stellar cusps.

Surprisingly, no unambiguous observational evidence for the existence (or not) of a stellar cusp around Sgr A* has been presented so far. While the first high angular resolution observations at an $8 \mathrm{~m}$ telescope appeared to indicate the existence of a stellar cusp (Genzel et al. 2003; Schödel et al. 2007), it was later realised that the star counts within about $0.5 \mathrm{pc}$ of Sgr A* were contaminated by a significant number of young, and therefore dynamically unrelaxed, stars. When omitting the young stars, the projected stellar density of giants appears almost flat, core-like, within a few $0.1 \mathrm{pc}$ of Sgr A* (Buchholz et al. 2009; Do et al. 2009; Bartko et al. 2010). Observations of the stellar surface brightness from old stars also appeared to indicate a possibly core-like structure (Fritz et al. 2016). These findings led to the missing cusp problem and have given rise to a large number of theoretical papers, trying to explain its absence. The hypotheses reach from a very long relaxation time (Merritt 2010), over the destruction of the envelopes of giants - thus rendering them invisible - via stellar collisions, which cannot fully explain the observations (Dale et al. 2009) or a fragmented gaseous disc, which can (Amaro-Seoane \& Chen 2014). Other explanations involve the apparent stellar structure arising from subsequent epochs of star formation and/or the accumulation of stellar remnants near Sgr A* (e.g. Löckmann et al. 2010; Aharon \& Perets 2015).

When evaluating the observational evidence, it is, however, of utmost importance to be aware of its limitations. Firstly, due to the extreme interstellar extinction towards the GC (e.g. Nishiyama et al. 2009; Schödel et al. 2010; Fritz et al. 2011), observations need to be performed in the near-infrared (NIR). A second requirement for observing the GC is to use an angular resolution as high as possible to overcome the extreme source crowding. Here we use the adaptive optics (AO) assisted NIR camera NACO installed at the ESO VLT. Imaging data at $H$ and $K_{\mathrm{s}}$ are used to be able to estimate extinction and - by the same means - to exclude foreground stars.

Because of these observational difficulties, our knowledge about the stellar population at the GC is limited to the brightest few percent of stars: a few million-year-old hot post main sequence (MS) giants and MS O/B stars (the latter being already at the faint limit of spectroscopic capabilities), on the one hand, and, on the other hand, giants with luminosities equal to or higher than RC stars. In fact, the typical spectroscopic completeness reaches only about $K_{\mathrm{s}}=15.5$ stars and thus only half the RC (see Do et al. 2009; Bartko et al. 2010; Do et al. 2015; Støstad et al. 2015; Feldmeier-Krause et al. 2015). Studies of the stellar surface density at the GC have so far been dominated by RC stars and brighter giants. The only recent study that focussed on the light from stars fainter than this limit did indeed find a cusp-like structure within $5^{\prime \prime} / 0.2 \mathrm{pc}$ of Sgr A* (Yusef-Zadeh et al. 2012). Hence, we have only observed the tip of the iceberg, which may not be representative for the overall, underlying structure.

This work continues similar efforts carried out by Genzel et al. (2003) and Schödel et al. (2007). As experiments are repeated, both their accuracy and precision tend to increase because of factors such as improvements in observational techniques, increasing know-how on data reduction and analysis, progress in theory and interpretation, and an increasing amount of data. The novel aspects of this work are, in particular, the stacking of high quality images with a large field-of-view (FOV) to reach fainter completeness limits in the most crowded regions near Sgr A*, an extension of high angular resolution data to a larger field of about $1.5^{\prime} \times 1.5^{\prime}$, improvements in data reduction (rebinning of the images, removal of systematic noise from detector electronics), and analysis (improved PSF fitting with use of noise maps and a spatially variable PSF, explicit consideration of systematic uncertainties caused by choice of parameters in the PSF fitting code). Finally, more explicitly than in previous work - and because our deeper data allow us to do so - we focus on clearly delimited stellar brightness ranges in order to minimise the mixing of different stellar populations. We add new data on the stellar distribution at projected radii $R \gtrsim 2 \mathrm{pc}$ from the literature (Schödel et al. 2014a; Fritz et al. 2016) to facilitate the interpretation of the data and the derivation of the 3D density structure of the stars near the massive black hole.

\section{Data reduction and analysis}

\subsection{Basic reduction}

We use $H$ and $K_{\mathrm{s}}$-band data obtained with the S27 camera $\left(0.027^{\prime \prime}\right.$ pixel scale) of VLT/NACO. The AO was locked on the NIR bright supergiant GCIRS 7 that is located about 5.5" north of Sgr A*. The data used are summarised in Table 1. All data were acquired with a similar four point dither pattern, roughly centred on Sgr A*, with the exception of the data from 11 May 2011, which covered a shallow, but wider mosaic with a $4 \times 4$ dither pattern, centred on Sgr A*. Preliminary data reduction was standard, with sky subtraction, bad pixel removal and flat fielding. Subsequently, a simple shift-and-add (SSA) procedure was applied to obtain final images. A quadratic interpolation with a rebinning factor of two was used because tests showed that this improved the photometry and reduced residuals in point spread function (PSF) fitting, in particular since the S27 pixel scale barely samples the angular resolution, which was roughly 0.06 " FWHM for all images. Along with the mean SSA images we also created noise maps that contain the error of the mean of each pixel in the SSA images.

\subsection{Pattern removal}

The images from the individual epochs contained horizontal stripe patterns from the detector electronics. These horizontal stripes can be detrimental for source detection because they may either mask faint sources or be deblended into rows of stars by the PSF fitting program. It is therefore important to remove 


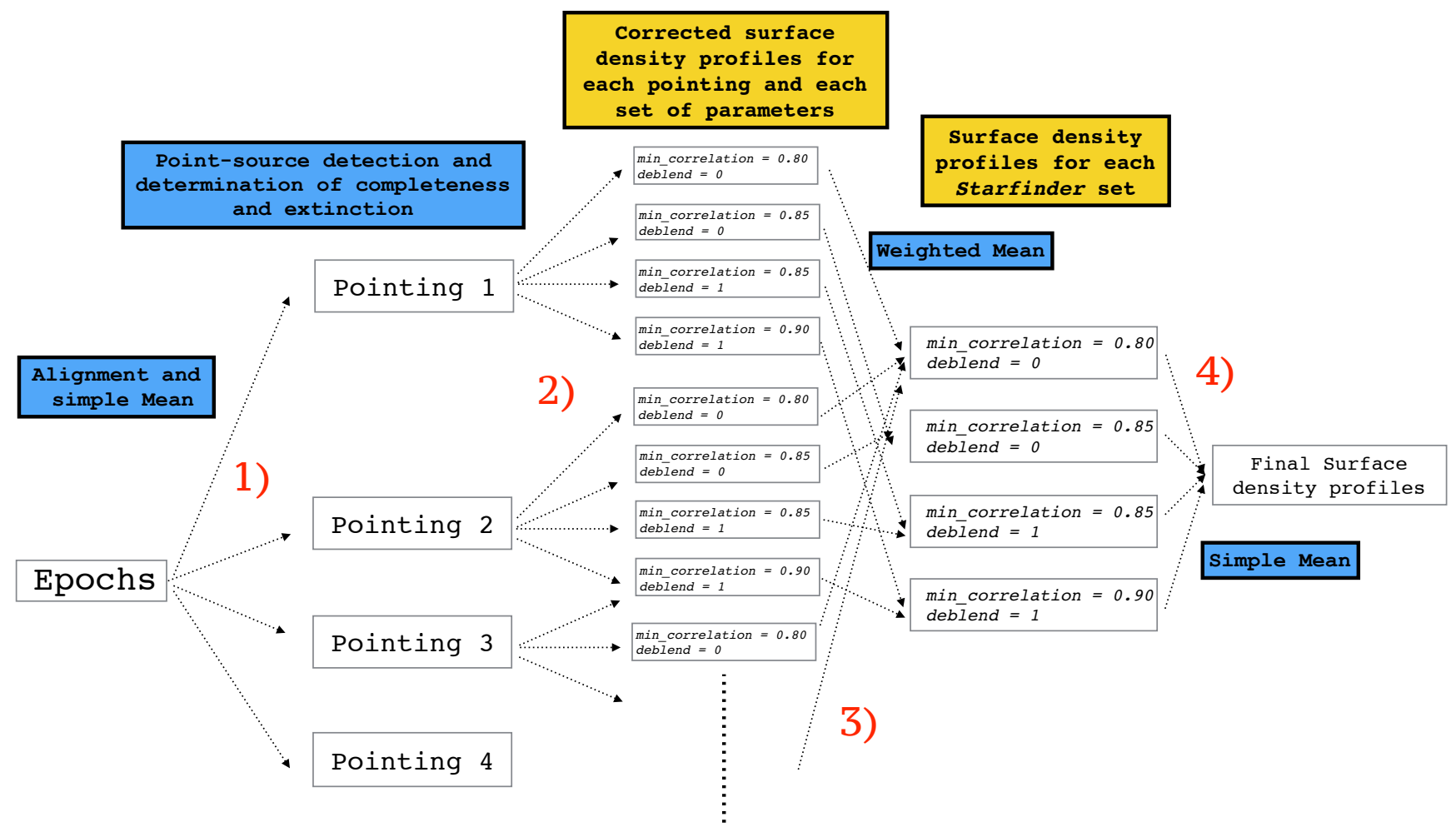

Fig. 1. Scheme of the procedure to compute the surface density profiles in the deep mosaic. The four pointings are analysed independently to avoid distortion problems. In step (1), the images from the different epochs for each of the four pointings are combined. In step (2), we analysed the photometry for the four pointings with four different values of the Starfinder parameter, determined the source detection completeness, computed the extinction map. Tables with the photometric and astrometric parameters for the point source detections, for each pointing and StarFinder parameter set are available at the CDS. Finally, we computed the extinction- and completeness-corrected surface density profiles. In the step (3), we computed the surface density profile for all Starfinder parameter sets by combining the results from the four pointings. In step (4), the surface density profiles obtained for each Starfinder set are mean-combined.

Table 1. Details of the imaging observations used in this work.

\begin{tabular}{llllll}
\hline \hline Date $^{a}$ & $\begin{array}{l}\lambda_{\text {central }} \\
{[\mu \mathrm{m}]}\end{array}$ & $\begin{array}{l}\Delta \lambda \\
{[\mu \mathrm{m}]}\end{array}$ & $N^{b}$ & NDIT $^{c}$ & $\begin{array}{l}\text { DIT }^{d} \\
{[\mathrm{~s}]}\end{array}$ \\
\hline 09 May 2010 & 1.66 & 0.33 & 4 & 64 & 2 \\
17 May 2011 & 2.18 & 0.35 & 4 & 9 & 2 \\
09 Aug. 2012 & 2.18 & 0.35 & 8 & 60 & 1 \\
11 Sep. 2012 & 2.18 & 0.35 & 8 & 60 & 1 \\
12 Sep. 2012 & 2.18 & 0.35 & 8 & 60 & 1 \\
\hline
\end{tabular}

Notes. ${ }^{(a)}$ UTC date of beginning of night. ${ }^{(b)}$ Number of (dithered) exposures. ${ }^{(c)}$ Number of integrations that were averaged on-line by the read-out electronics. ${ }^{(d)}$ Detector integration time. The total integration time of each observation amounts to $N \times$ NDIT $\times$ DIT.

them. We proceeded as follows: we used the StarFinder program to detect and subtract robustly detected point sources from each image (conservative settings of the StarFinder parameters: min_correlation $=0.85$ and deblend $=0$ ) and to fit the diffuse emission (from unresolved stars or dust and gas in the interstellar medium). The latter was fitted with an angular resolution of about $0.25^{\prime \prime}$, a non-critical value that just needs to be large enough to remove the variable background due to unresolved stellar emission and small enough to roughly correspond to the size of diffuse, unresolved structures in the mini-spiral (see $\mathrm{Pa}$ per II). While fitting of the diffuse background is important in this procedure, the exact choice of its variability scale is not. It can easily be chosen to be a factor 2 larger or smaller.
The resulting residual images, i.e. image minus diffuse emission minus point sources, were then dominated by small-scale (on the order a few pixels width) random and systematic noise. We determined the pattern of horizontal stripes induced by the electronics through median smoothing each row of pixels with a median box width of about 2.7", corresponding to 200 pixels (in the rebinned images). This pattern was then subtracted from the SSA images. We could thus remove most of the systematic noise without introducing any significant bias on the point sources or on the diffuse emission because most stars had already been subtracted and because the median smoothing box was a factor of a few to ten larger than the scales of the diffuse emission, of the size of PSF residuals, or of faint, unresolved sources. Finally, after having cleaned the images of each epoch, they were combined to a deep mean image (see next section). This last step further reduced any remaining systematics. To be conservative, we used the noise maps derived from the uncleaned images. Figure 2 shows details of $K_{\mathrm{s}}$-images to illustrate the effect of the systematic readout noise and the improvement after removing it. Figure 1 shows an outline of the procedure that we followed after this basic reduction.

\subsection{Alignment and stacking}

We treated each of the four pointings towards Sgr A* independently to avoid problems arising from camera distortions near the edges of the NACO S27 camera's field (see Trippe et al. 2008; Schödel et al. 2009). The final images from all epochs were aligned with the one from 09 August 2012 via a polynomial 

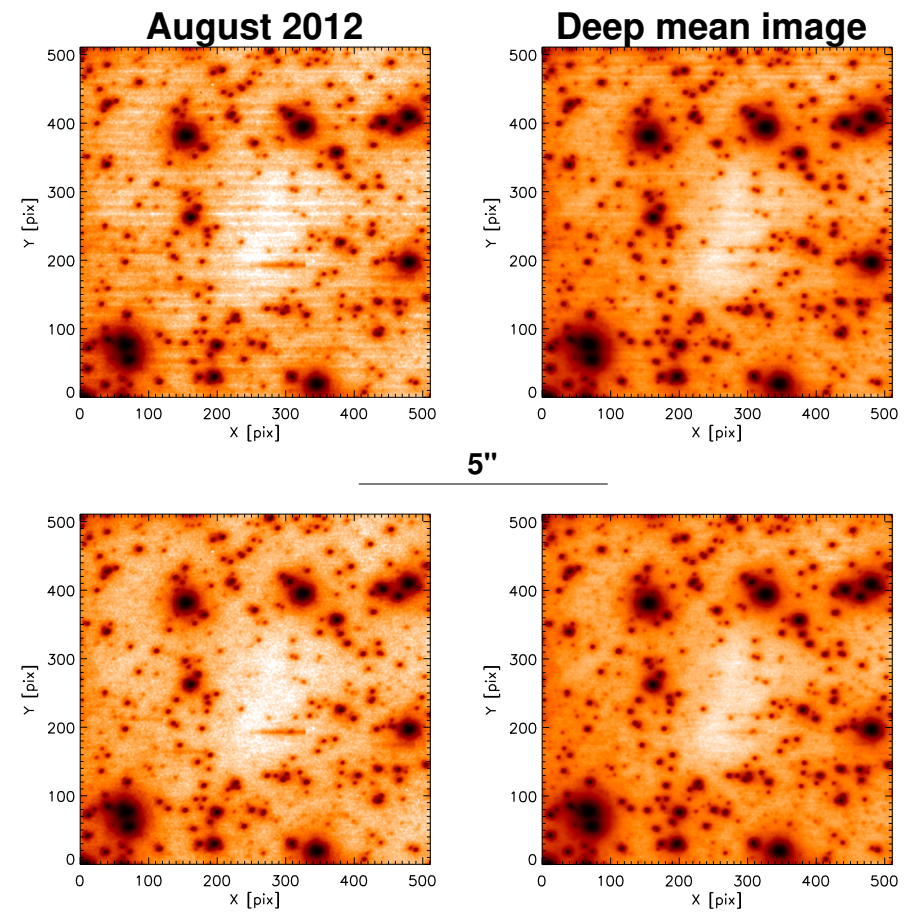

Fig. 2. Cleaning of horizontal stripes (systematic readout noise). Upper left: detail of August $2012 K_{\mathrm{s}}$-band image. Lower left: as upper left, but cleaned. Upper right: detail of deep, mean combined $K_{\mathrm{s}}$-band image when the input images have not been cleaned. Lower right: detail of deep, mean combined $K_{\mathrm{s}}$-band image after cleaning of the input images. The displayed field is located about $12.0^{\prime \prime}$ and $1.7^{\prime \prime}$ north of Sgr A*. The colour scale is logarithmic and identical for all images.

fit of degree one (IDL POLYWARP and POLY_2D procedures). The parameters of the latter were determined via an iterative fit using lists of detected stars in the image. The images were combined in a simple mean and the corresponding noise maps were quadratically combined (step 1 in Fig. 1).

A possible concern in this stacking procedure is the use of different observing epochs because of the large proper motions of the stars in the GC. At the distance of the GC (here assumed as $8 \mathrm{kpc}$, see, e.g. Genzel et al. 2010; Meyer et al. 2012), a velocity of about $40 \mathrm{~km} \mathrm{~s}^{-1}$ on the plane of the sky corresponds to a proper motion of one milli-arcsecond per year. A displacement by one pixel of the NACO S27 camera per year therefore corresponds to a velocity $>1000 \mathrm{~km} \mathrm{~s}^{-1}$. Since we are not interested in high precision astrometry or photometry, this effect is therefore negligible for our data, except possibly a small number of very fast moving stars within $\sim 0.1^{\prime \prime}$ of Sgr A*, which is not relevant to the problem and scales addressed in this paper.

\subsection{Source detection}

Point source extraction was carried out with the PSF fitting program StarFinder (Diolaiti et al. 2000). Since the images cover areas similar to or larger than the isoplanatic angle at $K_{\mathrm{s}}$-band, care was taken to deal with the spatial variability of the PSF. We parted the images into sub-fields of approximately $10.5^{\prime \prime} \times 10.5^{\prime \prime}$ size. Subsequently, ten of the brightest, most isolated stars in each sub-field were used for an iterative extraction of a local PSF (similar to what was done in Schödel et al. 2010). Because of variable extinction and source density, not all sub-fields contained PSF reference stars of similar brightness, which would lead to a systematic change of the zero point across the field. Also, not taking into account the extended seeing halo from the light that could not be corrected by the AO, can lead to an enhanced detection of spurious faint stars near bright stars. As remarked by Schödel (2010), the seeing halo is affected in a rather minor way by anisoplanatic effects. We therefore used the brightest star in the field, GCIRS 7, to estimate the seeing halo. The local PSFs were masked beyond radii of about $0.3^{\prime \prime}$, up to which they could be reliably determined. Then they were matched to the seeing halo (using a least-squares fit to determine flux offsets and normalisation factors). Thus, we could create local PSFs that avoided large systematic photometric effects across the field. Some tests (similar to what was done in Schödel 2010) showed that we could constrain the systematic photometric effects from the variability of the PSF to a few percent across the field.

In PSF fitting we have to walk a thin line between achieving an almost complete detection of sources while, at the same time, avoiding to pick up spurious ones, which can arise, in particular, close to bright stars or due to systematic effects from the detector electronics. We visually verified that taking the PSF seeing halos into account, along with the use of our SSA noise maps, effectively suppressed the detection of spurious sources near bright stars (see also Schödel et al. 2013). The PSF halos include effects such as diffraction spikes and static speckles. Our empirical noise maps seemed to deal well with suppressing the detection of spurious sources near bright stars.

Finally, since there can be no absolute certainty in the reliability of source detection, we also repeatedly analysed the images with different values of the StarFinder parameters that dominate the probability of source detection (for a fixed detection threshold, which was chosen as $3 \sigma$ in all cases). These parameters are min_correlation and deblend. For the minimum correlation value we chose $0.8-0.9$, always more conservative than the standard value of 0.7 , the default value of StarFinder. The key word deblend can be set to deblend close sources. While deblending can be very useful, it can lead also to the detection of a significant number of spurious sources in a crowded field. We included measurements with and without setting this keyword. We used the following four combinations of min_correlation and deblend: $[0.80,0],[0.85,0],[0.85,1]$, and $[0.90,1]$ (step 2 in Fig. 1).

\subsection{Photometric calibration and source selection}

Finally, the photometry was calibrated with the stars IRS 16C, IRS 16NW, and IRS 33N (apparent magnitudes $K_{\mathrm{S}}=9.93,10.14,11.20$ and $H=11.90,12.03,13.24$, see Schödel et al. 2010). The uncertainty of the zero points was a few percent. We note that for the purposes in this paper we do not require any high accuracy and high precision photometry and astrometry.

Almost all stars in the field have intrinsic colours $-0.1 \leq$ $H-K \leq 0.3$ (see, e.g. Do et al. 2009; Schödel et al. 2010). The mean colour due to reddening is $H-K \approx 2.1$. We excluded all stars with $H-K<1.5$ as foreground stars. We also excluded spectroscopically identified young stars from our final star list (Do et al. 2009; Bartko et al. 2010). Subsequently, we created an extinction map, by using the 20 stars nearest to each point. The resulting map is similar, to within the uncertainties, to the one presented in Schödel et al. (2010). 

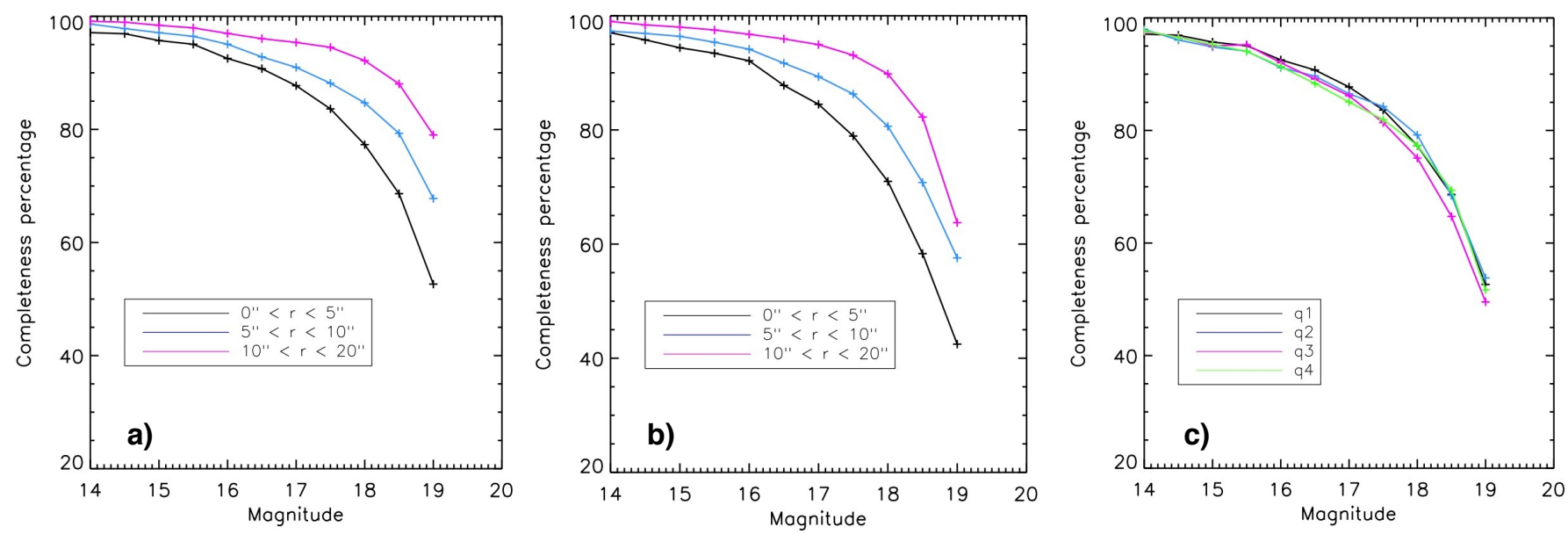

Fig. 3. Completeness of the star counts in the deep NACO $K_{\mathrm{s}}$ images. a) Completeness in pointing 1 for different projected distance ranges from Sgr A*, for min_correlation $=0.80$ and deblend $=0$. b) As in a), but for min_correlation $=0.90$ and deblend $=1$. c) Completeness for all four pointings and within $5^{\prime \prime}$ of Sgr A*, for min_correlation $=0.80$ and deblend $=0$. The corresponding plots for other used combinations of min_correlation and deblend look very similar.

\subsection{Crowding and completeness}

We determined the source detection completeness in the $K_{\mathrm{S}^{-}}$ images through the technique of inserting and recovering artificial stars for each of the four pointings (the second step in Fig. 1). We used a magnitude step of $0.5 \mathrm{mag}$ and inserted the stars on a $0.5^{\prime \prime} \times 0.5^{\prime \prime}$ grid. With this relatively wide spacing we avoided artificially increasing the crowding. The grid was shifted several times to finally probe completeness on a dense $0.1^{\prime \prime} \times 0.1^{\prime \prime}$ grid (as done by Schödel et al. 2007). We used the respective local PSFs (see above). Subsequently, PSF fitting was carried out with StarFinder and a source was considered as detected if it was found within a magnitude range of $0.5 \mathrm{mag}$ of the input magnitude and within a distance of $0.054^{\prime \prime}$ of the input position (corresponding to 2 pixels of the S27 camera or roughly the angular resolution of the data). If a real star of a similar magnitude was already present within this distance to the grid point of an artificial star, then the artificial star was considered as detected. This latter point is critical to avoid bias because the relatively high density of artificially introduced stars would otherwise lead to non-detection of real sources and thus an over-estimation of incompleteness.

As mentioned above, to estimate the systematic errors induced by either the non-detection of real sources or the detection of spurious sources, we repeated the source detection and completeness determination for the following combinations of the StarFinder parameters: min_correlation $=0.80,0.85,0.85,0.90$ and deblend $=0,0,1,1$ (each value in the first list corresponds to the value with the same index in the second list). In Fig. 3, we show the values of completeness for two of these cases and for different projected distance ranges from Sgr A*. The differences between the different choices of parameters are generally small, on the order of a few percentage points, except for the faintest magnitudes, where the differences are somewhat more pronounced. Also, we can observe the expected general trend of less completeness for fainter magnitudes and in the more crowded areas near Sgr A*. Finally, Fig. 3 also shows that the differences of completeness between the four pointings are small. For all cases, we found that source detection was, at all projected distances, at least $50 \%$ complete for magnitudes $K_{\mathrm{s}} \leq 18.5$ (Step 2 in Fig. 1).

\subsection{Extinction}

We used the $H-K_{\mathrm{s}}$ photometry and the intrinsic small colours of stars at these bands to create an extinction map for each of the four pointings (the second step in Fig. 1), with the same method as applied in Schödel et al. (2010). We do not consider stars with $H-K_{\mathrm{s}}<1.5$ because we consider them as foreground stars. Neither do we consider stars with $H-K_{\mathrm{s}}>3.0$ because they may either be background stars or intrinsically reddened objects (in any case, their number is very small, see Fig. 4 in Schödel et al. 2010). Median stellar colours were obtained from the individual colours of the 20 nearest stars at each position and the extinction was then calculated as in Schödel et al. (2010), assuming $A_{K_{\mathrm{s}}} \propto$ $\lambda^{-2.2}$.

On the one hand, the extinction map was used to correct the individual stellar magnitudes for differential extinction. On the other hand, we applied the methodology of Chatzopoulos et al. (2015b) to compute the stellar detection completeness variation caused by variable extinction: we modelled the luminosity function (LF) by taking the product of a power-law stellar LF and an error function that represents the completeness function, as in expression (2) in Chatzopoulos et al. (2015b). The approximation of the LF with a power-law - which ignores the presence of the RC bump - does not introduce any significant error because our data are sensitive enough to reach well below the RC bump over the entire field and because including the RC bump would only have a minor effect as shown by Chatzopoulos et al. (2015b). First, we measured the observed KLF for each pointing and each StarFinder parameter set (excluding a radius of about $5^{\prime \prime}$ around $\mathrm{Sgr} \mathrm{A}^{*}$, where the KLF is more incomplete because of crowding). We computed the power law for each case. Finally, we used these power-laws, combined it with the measured local extinction and computed the corresponding local correction factors according to Eq. (5) in Chatzopoulos et al. (2015b), but using the approximation of a single extinction screen, i.e. no variability of $A_{K_{\mathrm{s}}}$ along the line-of-sight. Since we approximate the LF with a power law, the equation takes on the form $p=L\left(-\Delta A_{k}\right)=10^{-\gamma * \Delta A_{k}}$ (see Chatzopoulos et al. 2015b), where $p$ is the reduction factor for the number of locally detected stars, $\Delta A_{k}$ is the difference between the local extinction and the mean extinction over the field, and $\gamma$ is the power law index of the luminosity function. If the local extinction is lower than the mean 


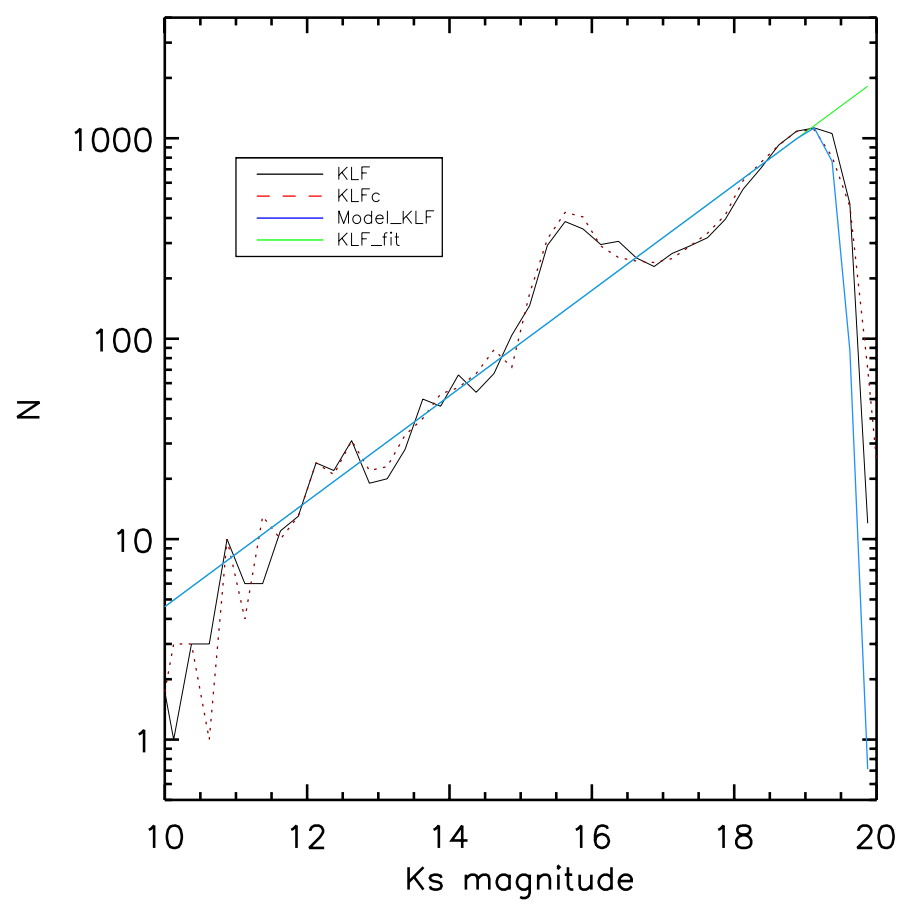

Fig. 4. The $K_{\mathrm{S}} \mathrm{LF}$ in pointing 1 for min_correlation $=0.8$ and $d e$ blend $=0$ if shown as a black line. The dotted red line is the $K_{\mathrm{s}} \mathrm{LF}$ after correcting the magnitude of each star for differential extinction. The green line shows a power law fit to the bright stars $11<K_{\mathrm{s}}<14.5$ with power law index of $0.26 \pm 0.02$. The blue line shows the effect of the completeness function according to Eq. (2) in Chatzopoulos et al. (2015b).

extinction, then $p>1$, and if the local extinction is higher than the mean extinction, then $p<1$.

We apply the correction factor $1 / p$ to each detected star. In Fig. 4 we show the $K_{\mathrm{s}} \mathrm{LF}$ for pointing 1 , for min_correlation $=0.8$ and deblend $=0$, along with the LF corrected for differential extinction, a power law fit to the stars $11<K_{\mathrm{s}}<14.5(\gamma=0.26 \pm 0.02$, similar to the value obtained in Schödel et al. 2010), and the completeness function (blue line), as defined by Chatzopoulos et al. (2015b). For the latter, we use $m_{0}=19.4$ and $\sigma=0.2$ because these values approximate our $K_{\mathrm{s}} \mathrm{LF}$ well. These values are different from those used in Chatzopoulos et al. (2015b) because our data are significantly deeper than theirs. In Fig. 5 the percentage reduction in observed stars versus projected radius is represented for the detected stars in pointing 1 , for min_correlation $=0.8$ and deblend $=0$. One can see that extinction is, on average, higher at larger distances from $\mathrm{Sgr} \mathrm{A}^{*}$, but that the effect of differential extinction on completeness is relatively minor, typically $<10 \%$ and at most $20 \%$.

\subsection{Wide field}

The 2011 data are of excellent quality, but relatively shallow. On the other hand, there are 16 pointings that cover a field of about $1.5^{\prime} \times 1.5^{\prime}$, compared to the smaller fields of about $40^{\prime \prime} \times 40^{\prime \prime}$ covered by the other NACO observations. The 2011 observations are therefore ideally suited to extend the sensitive central observations with high angular resolution, albeit somewhat shallower, data out to larger distances (Fig. 6). Although the wide field data are shallow, in the sense of small total exposure time, they are used here at large projected radii, $R$. Since crowding, not

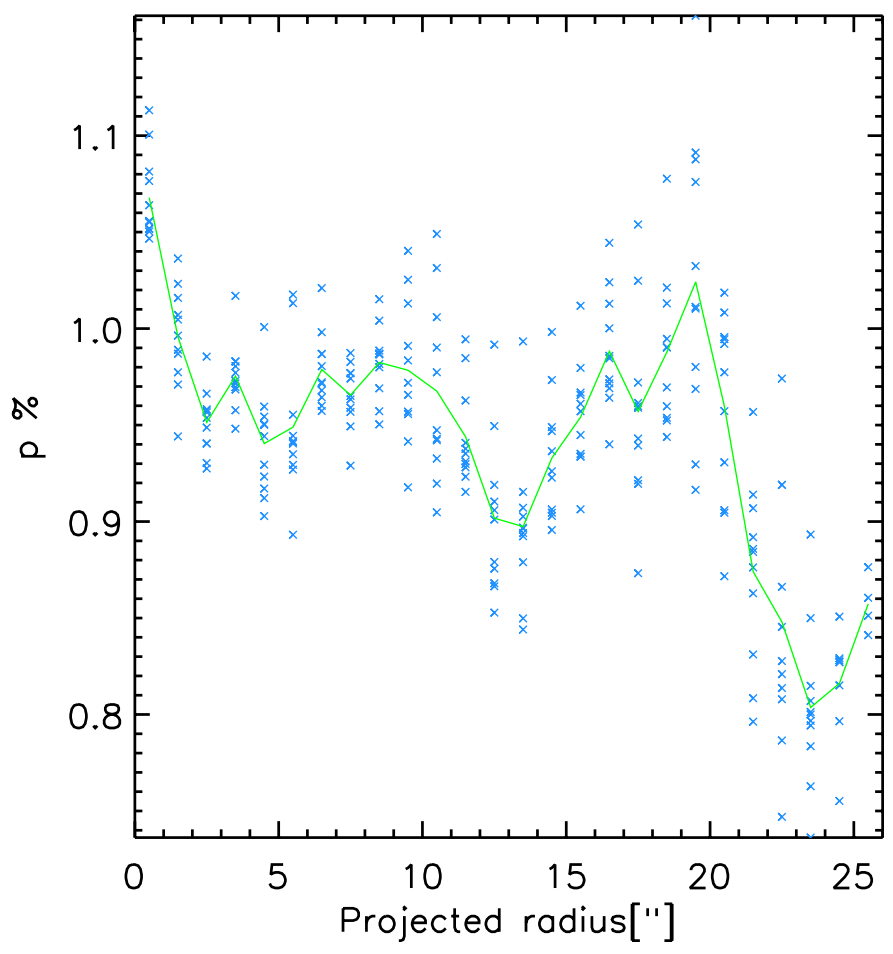

Fig. 5. Relative detection frequency due to extinction versus projected distance to $\mathrm{Sgr} \mathrm{A}^{*}$, in pointing 1 for min_correlation $=0.8$ and $d e$ blend $=0$. The blue crosses give the values of $p$ for each magnitude bin. The green line represents the mean of the $p(\%)$ considering detected stars at the same distance from Sgr A*. We can observe that for close distances to $\operatorname{Sgr} \mathrm{A}^{*} p(\%)$ is higher than for large distances, as we expected, because the extinction near $\mathrm{Sg} \mathrm{A}^{*}$ is lower.

integration time, is the main factor that limits source detection at the GC, the $50 \%$ completeness limit of the wide field data is still as low as $K_{\mathrm{s}} \approx 18.5$ at $R>0.5 \mathrm{pc}$. They are therefore ideal to be combined with our deep data at smaller $R$.

In order to deal with the distortions of the NACO S27 camera (see, e.g. Trippe et al. 2008; Schödel et al. 2009) we aligned each pointing of the NACO mosaic with a reference frame created from positions measured in HST WFC3 observations of the same field (Dong et al., in prep.). We apply variable PSF fitting as we explain in Sect. 2.4. In this case, min_correlation $=0.8$ and deblend $=0$ were selected for the StarFinder parameters.

\section{3. $K_{\mathrm{s}}$-luminosity function}

The goal of this study is to investigate the existence of a stellar cusp at the GC. This requires us to select stars old enough to have undergone dynamical relaxation. The relaxation time at the GC is roughly a few Gyr (e.g. Alexander 2005, 2011). We specifically exclude all spectroscopically identified early-type, i.e. young and massive, stars from our sample (using the data of Do et al. 2013). Unfortunately, spectroscopic stellar classification is limited to stars of about $K_{\mathrm{s}} \leq 15.5$ at the GC. For fainter stars, we can only use their luminosity as a proxy for their type. Figure 16 of Schödel et al. (2007) illustrates the LF, mean masses, and old star fractions for stars of different magnitudes at the GC, assuming continuous star formation at a constant rate over the last $10 \mathrm{Gyr}$. It shows that we can probe old ( $\gtrsim 1 \mathrm{Gyr}$ ), low-mass stars in the range $15 \lesssim K_{\mathrm{s}} \leq 16$. This is the RC, which dominates all previous star density measurements. The fraction of old stars rises again above $\sim 50 \%$ for stars $K_{\mathrm{s}}>17.5$, 


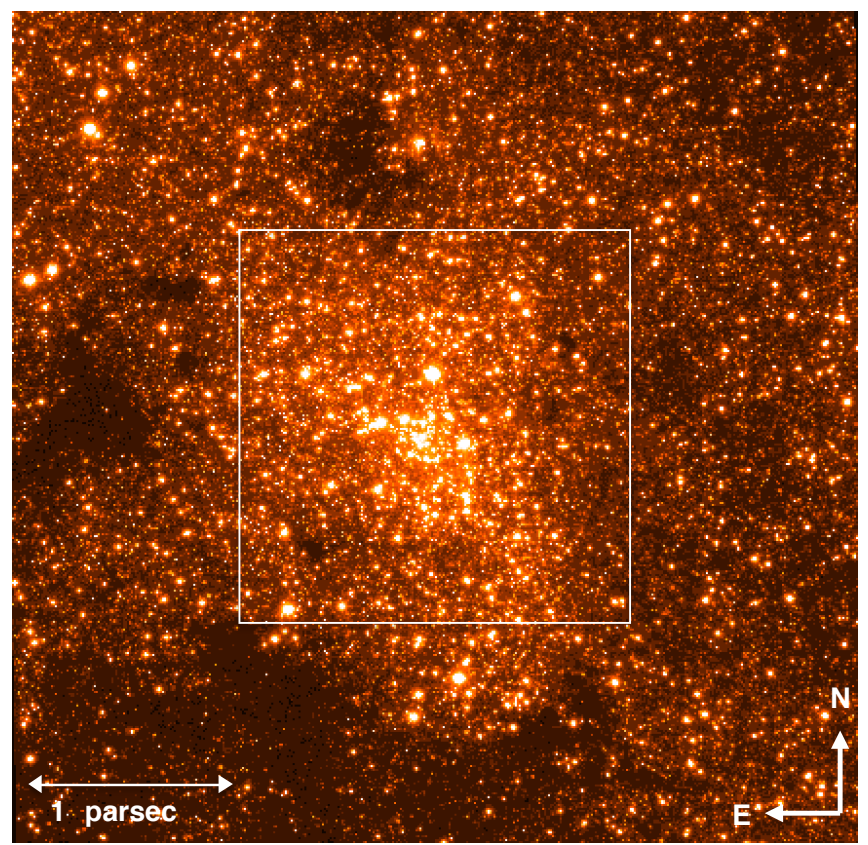

Fig. 6. Wide-field mosaic the observations from 11th May 2011. The field-of-view is $1.5^{\prime} \times 1.5^{\prime}$. The field of about $40^{\prime \prime} \times 40^{\prime \prime}$ that corresponds to the deep imaging data is marked by a white square.

reaching practically $100 \%$ at $K_{\mathrm{s}} \approx 18$. Therefore, in this study, we focus on the magnitude ranges $15 \leq K_{\mathrm{s}} \leq 16$, the RC, and $17.5 \leq K_{\mathrm{s}} \leq 18.5$, i.e. the faintest stars accessible by our data.

We show the $K_{\mathrm{s}}$ luminosity function (KLF) determined from our deep mosaic in Fig. 7. The KLFs corresponding to the four different StarFinder parameter settings are shown. The KLF derived in this work is about one magnitude deeper compared to previous work (Fig. 10 in Schödel et al. 2007). This is a decisive advantage. When we want to probe the existence of a dynamically relaxed stellar cusp around $\operatorname{Sgr} \mathrm{A}^{*}$, we need to focus on stars that are at least several Gyr old. As shown in the illustrative Fig. 16 in Schödel et al. (2007), the only magnitude range where this was previously possible was around the RC $\left(15.25 \leq K_{\mathrm{s}} \leq 16.25\right)$. Now, with the deeper data from our new analysis, we can probe another, fainter magnitude range with a high fraction of old stars $\left(17.5 \leq K_{\mathrm{s}} \leq 18.5\right)$. Also, the stars in these two brightness ranges have similar masses, which lets us expect a similar surface density distribution. We discuss in Sects. 5.2 and 5.3 the different populations that we can expect in the faintest range of stars, based on the latest determined star formation history for the GC, and the possible contamination of our star counts by stars too young to be dynamically relaxed.

\section{The 2D density of old stars in the GC}

In order to analyse the surface density of stars at the GC, we assume that the underlying spatial distribution of the stars in the central parsec is spherically symmetric. Although the nuclear cluster is flattened, a spherical approximation should be acceptable at projected radii $R \lesssim 2$ pc because the difference between the density profiles along the orthogonal directions of maximum difference is only on the order of 10-20\% in this region (see Schödel et al. 2014a; Fritz et al. 2016).

\subsection{Star counts in the central parsec}

We computed the azimuthally averaged stellar surface densities in annuli around $\mathrm{Sgr} \mathrm{A}^{*}$. It is important to choose a number of bins sufficiently large to capture the major features in the data while ignoring fine details due to random fluctuations. Following the studies of Knuth (2006) and Witzel et al. (2012) we first determine the best bin size. The dependence of the Relative Logarithmic Posterior Probability (RLP) on the bin number for pointing 1 is shown in Fig. 8. The maximum for the RLP for the star number is reached for 21 bins, and the best bin size is $1^{\prime \prime}$. We applied this methodology for all pointings, with similar results.

We computed extinction and completeness-corrected stellar surface densities for the stars detected in the different StarFinder runs and for the different pointings (the second step in Fig. 1). At this point we included the uncertainties of the different correction factors into the uncertainties of the stellar surface densities. For faint stellar magnitudes, we masked the regions with completeness below $30 \%$. We tested the effect of different masks, with completeness $<30 \%, 40 \%, 50 \%$ respectively, and found that the results did not vary significantly. Finally, we obtained the surface density profiles for each set of Starfinder parameters by combining the measurements on the four pointings in a weighted mean (the third step in Fig. 1). Finally, we combined the surface density profiles obtained with the four settings of the StarFinder parameters. Mean densities and standard deviations were computed and all uncertainties were quadratically combined (the last step in Fig. 1)

\subsection{Star counts beyond $20^{\prime \prime}$}

In order to study the stellar number density in a broader range of distances from $\mathrm{Sgr} \mathrm{A}^{*}$ we analysed the large mosaic image from the 2011 data. We did not apply any extinction and completeness corrections because the effect of the extinction correction is small and crowding does not pose any serious problem at $R>20^{\prime \prime}$ and with the high angular resolution data used here. We did, however mask all the regions occupied by the dark clouds that can be seen show in Fig. 6. Finally, we combined the data from the deep field and the wide field. The surface densities from the wide field data were scaled to the completeness and extinction corrected ones from the deep images in the overlap region from $R=10^{\prime \prime}-20^{\prime \prime}$.

Figure 9 shows the combined number density plots for stars in the magnitude range $12.5 \leq K_{\mathrm{s}} \leq 16.0$ (red) and $17.5 \leq K_{\mathrm{s}} \leq$ 18.5 (black) stars (right), along with simple power-law fits. The brighter magnitude interval was chosen to trace bright, old giant stars, based on current estimates of the star formation history in the central parsec (see Sect.5.2 and Fig. 11). The faint magnitude interval corresponds to the faintest stars that we detect with completeness higher than $50 \%$ across the field (see Sect. 2.6).

\subsection{Projected surface number density}

Simple power laws of the form $\Sigma(R) \propto R^{-\Gamma}$ were fit to the surface number densities, where $\Sigma$ is the surface number density, $R$ the projected radius, and $\Gamma$ the power-law index. We fitted the power laws to the data in different distance ranges. The corresponding power law indices and the $\chi^{2}$ values of the fits are listed in Table 2 and the data and one of the fits are shown in Fig. 9. All formal uncertainties from the fits were rescaled to a reduced $\chi^{2}=1$ here and in the rest of the paper. We observe that: (1) a simple power-law provides a better fit to the faint stars $\left(K_{\mathrm{S}} \approx 18\right)$ than to the bright giants; $(2)$ the value of $\Gamma$ depends on 

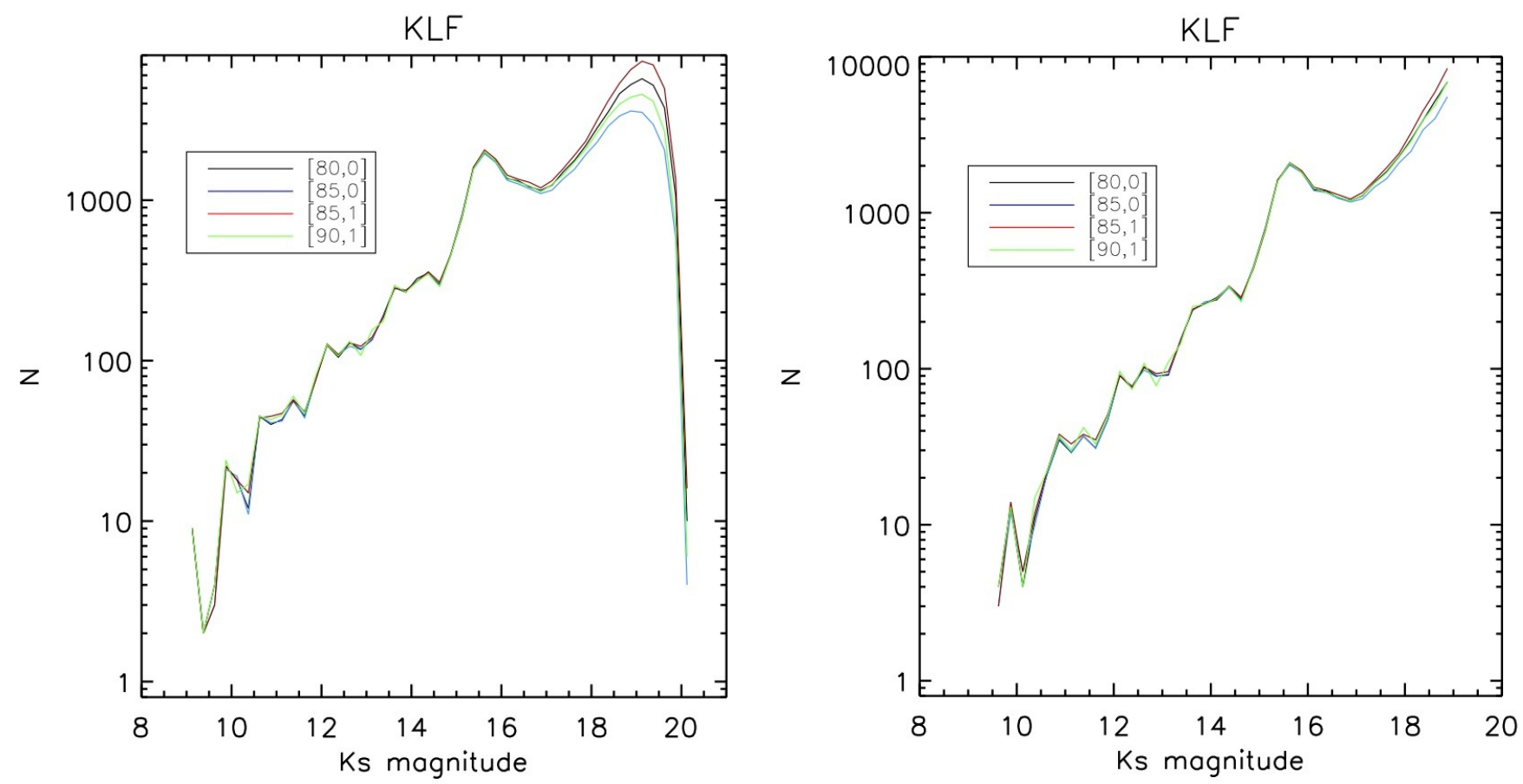

Fig. 7. KLF for the deep $K_{\mathrm{s}}$ mosaic. The different colours correspond to the different combinations of the values of min_correlation and deblend, as listed in the legend (see also Sect. 2.4). Left: raw KLF. Right: completeness- and extinction-corrected KLF.

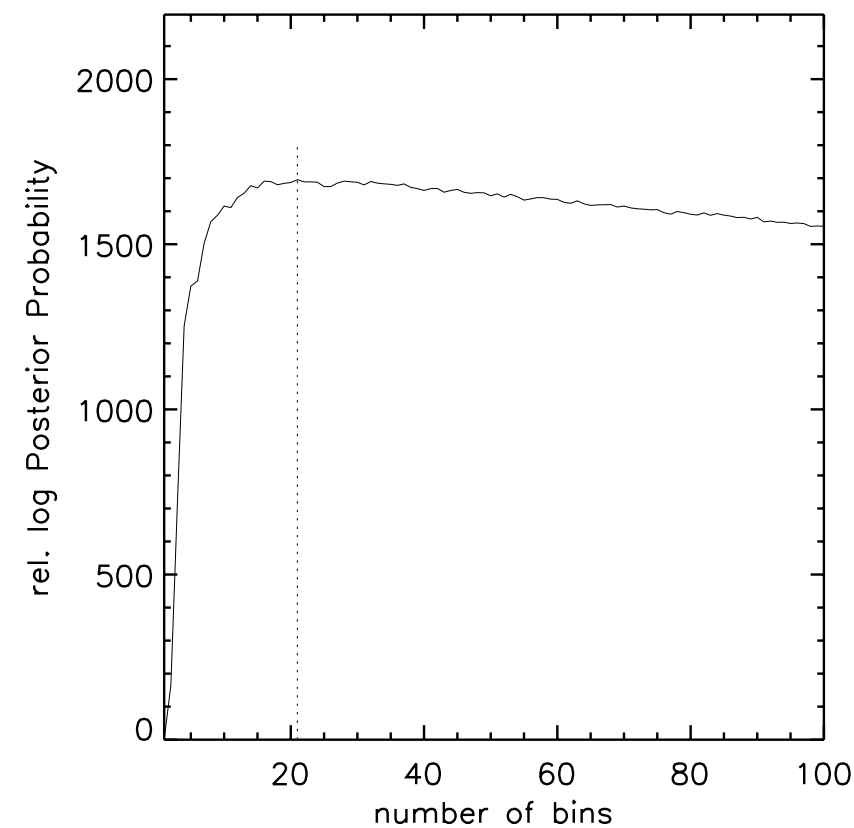

Fig. 8. Optimal binning. We show the RLP as a function of the number of bins. The maximum for the RLP is reached for 21 bins (red dotted line).

the range in $R$ used for the fitting, with a tendency for a steeper power-law at greater distances; (3) the data point at $R<1^{\prime \prime}$ lies below the fit in all cases. This region is the most crowded region with a possibly altered stellar population (the S-stars) and we omitted it therefore from our fits; (4) the giant stars show a flat, or even decreasing surface density at $R \lesssim 8^{\prime \prime}$, in agreement with what has been found before (Buchholz et al. 2009; Do et al. 2009; Bartko et al. 2010). They also display a significant dip

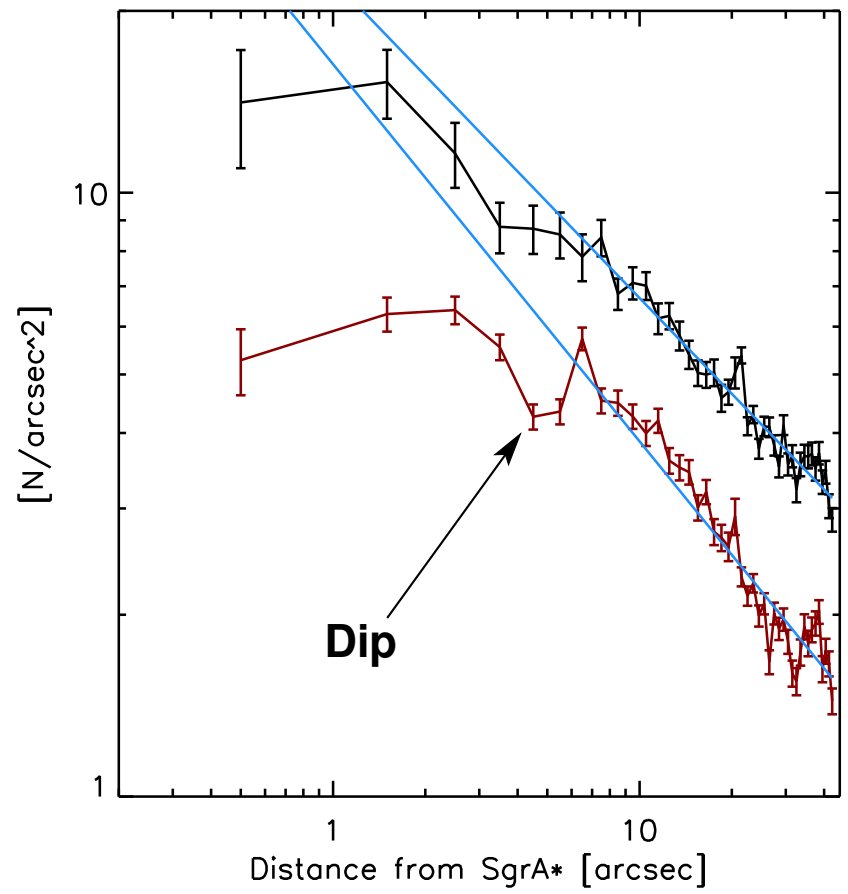

Fig. 9. Combined deep field plus wide field surface density plots for stars in the magnitude intervals $12.5 \leq K_{\mathrm{s}} \leq 16$ and $17.5 \leq K_{\mathrm{s}} \leq$ 18.5. The blue lines are simple power-law fits to the data at $0.2 \mathrm{pc} \leq$ $R \leq 1.0 \mathrm{pc}$. Tables with the stellar surface density data have been made available at the CDS.

around $R=0.2 \mathrm{pc}\left(5^{\prime \prime}\right)$ in the density profile and, possibly, an excess at $R \approx 7^{\prime \prime}$, that are also visible in the works of Schödel et al. (2007) and Buchholz et al. (2009).

The projected surface number density of the stars in the interval $17.5 \leq K_{\mathrm{s}} \leq 18.5$ can be described well by a single powerlaw. Its mean value and standard deviation, taking into account 
Table 2. Values of the power-law index, $\Gamma$, for the extinction and completeness-corrected surface density profiles.

\begin{tabular}{lllll}
\hline \hline ID & Fit range $(\mathrm{pc})$ & Magnitudes range $\left(K_{\mathrm{s}}\right)$ & $\Gamma$ & $\chi_{\text {reduced }}^{2}$ \\
\hline 1 & $0.04-0.5$ & $17.5-18.5$ & $0.36 \pm 0.04$ & 0.8 \\
2 & $0.04-0.5$ & $12.5-16.0$ & $0.24 \pm 0.02$ & 4.9 \\
3 & $0.2-1.0$ & $17.5-18.5$ & $0.53 \pm 0.03$ & 2.6 \\
4 & $0.2-1.0$ & $12.5-16.0$ & $0.62 \pm 0.02$ & 5.1 \\
5 & $0.04-1.0$ & $17.5-18.5$ & $0.47 \pm 0.02$ & 2.8 \\
6 & $0.04-1.0$ & $12.5-16.0$ & $0.45 \pm 0.01$ & 9.2 \\
7 & $0.5-1.5$ & $17.5-18.5$ & $0.50 \pm 0.03$ & 3.2 \\
8 & $0.5-1.5$ & $12.5-16.0$ & $0.73 \pm 0.03$ & 4.1 \\
9 & $0.5-2.0$ & $17.5-18.5$ & $0.50 \pm 0.03$ & 3.1 \\
10 & $0.5-2.0$ & $12.5-16.0$ & $0.66 \pm 0.03$ & 4.6 \\
\hline
\end{tabular}

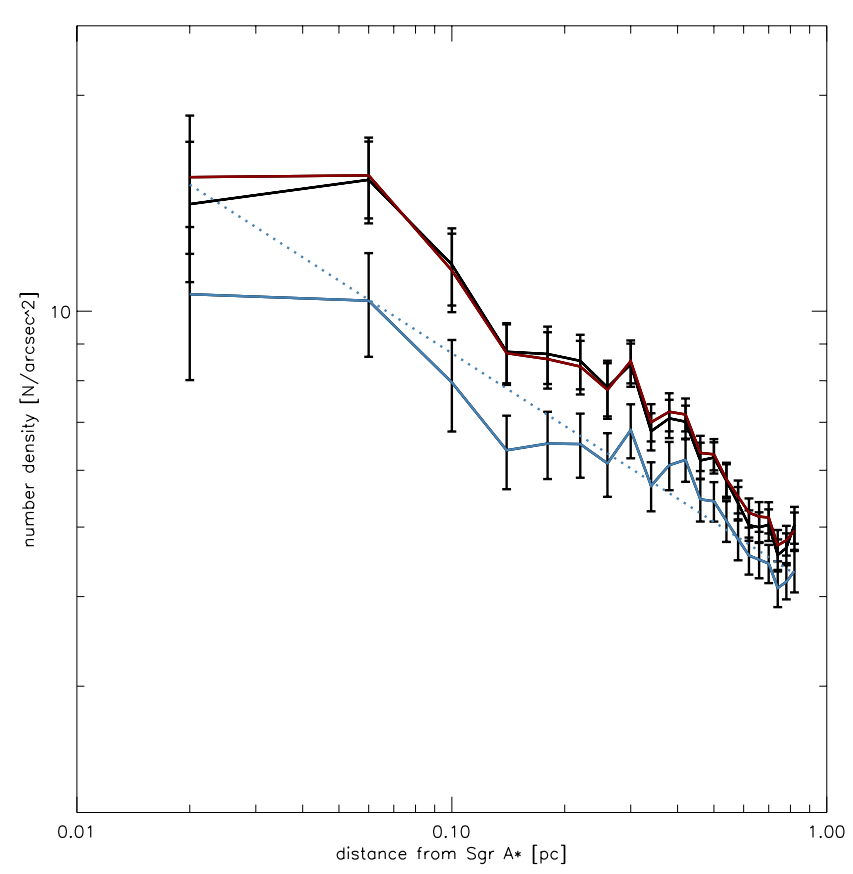

Fig. 10. Mean surface density profile for stars with $\left(17.5 \leq K_{\mathrm{s}} \leq 18.5\right)$, after averaging over the four runs with different Starfinder parameters. Blue: uncorrected data. Red: data corrected for crowding. Black: data corrected for crowding and extinction.

the different fitting radii in Table 2 is $\Gamma_{\text {faint }}=0.47 \pm 0.07$. The giants present a somewhat different picture: a single power-law provides only a good fit to the data at $R \gtrsim 8^{\prime \prime} / 0.24 \mathrm{pc}$. To take this into account, we exclude fit ID 2 from Table 2 and obtain a mean value of $\Gamma=0.62 \pm 0.12$.

\section{Discussion}

\subsection{Influence of the correction factors}

When determining the number density profile, we have to apply correction factors to compensate the effects of variable stellar crowding and interstellar extinction. Figure 10 shows the measured surface density profile for stars of magnitude $17.5 \leq K_{\mathrm{s}} \leq$ 18.5 detected in the deep mosaic without any correction, after applying the completeness correction for crowding, and after applying the completeness corrections for crowding and extinction. As we can see, the completeness correction steepens the profile somewhat. The extinction correction only introduces minor changes because the azimuthal averaging compensates most of the effects of differential extinction across the field. Fitting a simple power-law to the uncorrected data in the range $0.04 \mathrm{pc} \leq R \leq$ $1 \mathrm{pc}$, we obtain $\Gamma=0.33 \pm 0.03$ (for the fully corrected data we obtain $\Gamma=0.47 \pm 0.02$, see Table 2 ). The effect of crowding correction is almost negligible for the giants brighter than $K_{\mathrm{s}} \approx 16$. We note that the uncertainties of the crowding and extinction corrections are included in all error bars and will therefore be reflected in the formal uncertainties of the best-fit parameters.

For the wide field data we did not apply any extinction and completeness corrections because we lacked the necessary complementary wide-field $H$-band imaging data. In any case, the effect of the extinction correction on the number density is small and crowding does not pose any serious problem beyond $20^{\prime \prime}$. Hence, the wide field data are scaled to the deep data in the overlap region. We mask the regions occupied by the dark clouds in the wide field image (see Fig. 6) to compute the surface density profile. In summary, the applied correction factors, albeit necessary, do not significantly alter our results. This shows that our data are robust. We note, however, that the wide field image in Fig. 6 appears to show that extinction is higher at larger $R$, in particular to the west of $\operatorname{Sgr} \mathrm{A}^{*}$. Since we do not correct the magnitudes of the stars detected in the wide image for extinction, this may result in giants dropping out of the brightness bin considered here. This may explain why the projected surface density of the giants appears to show a slightly steeper decrease at large $R$ than the surface density of faint stars (Fig. 9).

\subsection{Age of tracer populations and possible contamination by $\sim 100$ Myr-old stars}

With an approximate magnitude of $K_{\mathrm{s}}=18$, the faintest stars in our sample are consistent with being (sub-)giants on the ascending branch or main sequence (MS) stars of $\sim 2.5 M_{\odot}$. They could also be pre-MS stars of a few solar masses or less (Lu et al. 2013). From what is known about the star formation history of the NSC we may expect that the majority of stars is old ( $~ 80 \%$ of the NSC's mass were formed $>5$ Gyr ago, according to Blum et al. 2003; Pfuhl et al. 2011) and that most of the faint stars in our sample are thus old, (sub-)giants.

However, there are two important caveats: (1) we know that a star formation event created on the order $10^{4} M_{\odot}$ of young stars in the region about $0.5 \mathrm{pc}$ around $\mathrm{Sgr}^{*}$ (Bartko et al. 2010; Lu et al. 2013; Feldmeier-Krause et al. 2015). Therefore contamination by pre-MS stars is possible. We will discuss this possibility in the next subsection; (2) there is evidence that the star formation rate in the central parsec was high about $100 \mathrm{Myr}$ ago (e.g. Blum et al. 2003; Nishiyama et al. 2016; Pfuhl et al. 2011). 


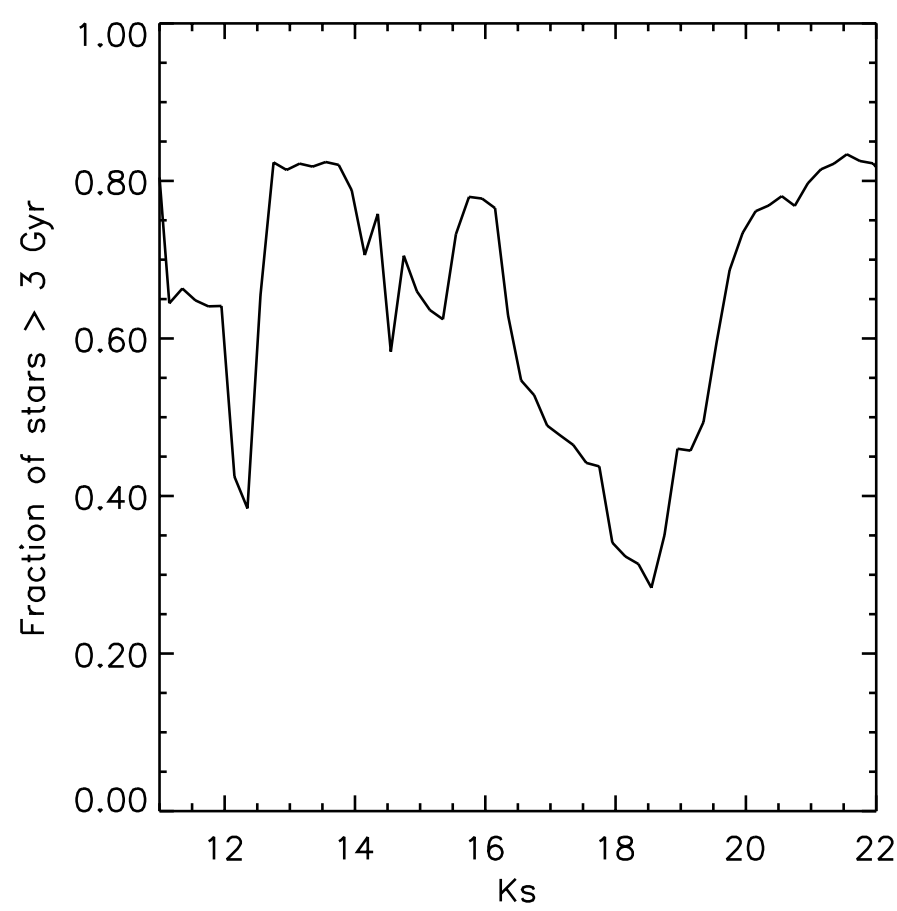

Fig. 11. Fraction of stars older than $3 \mathrm{Gyr}$ as a function of their observed $K_{\mathrm{s}}$-magnitude at the GC, computed based on the SFH derived by Pfuhl et al. (2011).

Using the star formation history (SFH) given by Eq. (3) in Pfuhl et al. (2011), we calculated the fraction of stars older than $3 \mathrm{Gyr}$ as a function of magnitude, as shown in Fig. 11. As we can see, contrary to what we assumed in Sect. 3 based on a model of constant star formation rate in the GC (Schödel et al. 2007), the magnitude interval around $K_{\mathrm{s}}=18$ may be dominated by relatively young, dynamically unrelaxed stars.

Unfortunately, currently there do not exist any adequate data on the age composition of the $K_{\mathrm{s}}=18$ stars and on the surface density of the potential different populations, which would allow us to consider an explicit correction for young stars. Depending on the exact properties and spatial distribution of these stars, the cusp signature could be enhanced or diminished. This is a source of systematics of unknown impact and needs to be investigated by future research. We note that the spectroscopic classification of $K_{\mathrm{s}}=18$ stars at the GC is beyond the reach of current instrumentation and may require telescopes of the $30 \mathrm{~m}$ class.

\subsection{Possible contamination by pre-MS stars}

Our primary goal in this study is to determine the spatial distribution of the old, relaxed stellar population at the GC. Care must therefore be taken to exclude young and therefore probably dynamically unrelaxed stars. We have excluded from the analysis all spectroscopically identified early-type stars from Do et al. (2013). Unfortunately, the limit of spectroscopic identification of early-type stars with current instruments is $K_{\mathrm{s}} \approx 16$ in the GC (with the exception of a few, very deep exposures of small fields that reached $K_{\mathrm{s}} \approx 17.5$, see Pfuhl et al. 2011). As argued in the previous section, the star formation event that took place a few Myr ago in the central $R \approx 0.5 \mathrm{pc}$ means that we have to consider explicitly the possibility that our stellar surface number densities are contaminated by pre-MS stars. As we discuss in Sect. 4.3 in Paper II, the possible contamination of the surface number density of $K \approx 18$ stars by young stars from the most recent,
5 Myr-old star formation can be relative less important than the contamination from other young or intermediate-age populations, with ages $\leq 3$ Gyr (see Fig. 7 in Paper II). While we cannot constrain the properties of this intermediate-age population due to lack of data, as explained in the preceding paragraph, we have more knowledge on the youngest stellar population and can thus consider its contaminating effect more explicitly.

To estimate the surface density of young stars from the most recent star formation event in the GC, we assumed a 5 Myr old cluster of mass $2.5 \times 10^{4} M_{\odot}$ of solar metallicity. From some experiments with different values we concluded that assuming somewhat different ages, masses, or metallicities will not change our conclusions significantly. We created different present-day model KLFs for this star formation event. On the one hand, we used the CMD 3.0 tool $^{1}$ (see Bressan et al. 2012; Chen et al. 2014; Tang et al. 2014) with a Kroupa IMF and the photometric system based on the works of Maíz Apellániz (2006) and Bessell (1990). On the other hand, we used the IAC-STAR tool (Aparicio \& Gallart 2004) to create KLFs with similar parameters, but with a different IMF, using, on the one hand, the extremely flat IMF $\mathrm{d} N / \mathrm{d} m \propto m^{-0.45}$ of Bartko et al. (2010) and, on the other hand, the steeper, but still top-heavy IMF $\mathrm{d} N / \mathrm{d} m \propto m^{-1.7}$ of Lu et al. (2013). We normalised with the value of 0.3 stars per square $\operatorname{arcsec}$ at $K \approx 15$ (Do et al. 2013; Lu et al. 2013) and computed the surface density at $R=2^{\prime \prime}$ assuming that the surface density of the young stars follows a power law of the form $\Sigma(R) \propto R^{-\eta}(\eta=0.93-1.1$, Bartko et al. 2010; Lu et al. 2013). The resulting KLFs for the young stars at $R=2^{\prime \prime}$ is shown in Fig. 12, where we also over-plot the KLF from our full data set, scaling with the surface number density of $K_{\mathrm{s}}=18$ stars at $R=2^{\prime \prime}$.

As was pointed out in previous work (e.g. Paumard et al. 2006; Bartko et al. 2010; Lu et al. 2013), the IMF of the most recent star formation event near Sgr A* appears to have been top-heavy. This is supported by our analysis of the KLF here: as can be seen in Fig. 12 the surface number density of young stars at $R=2^{\prime \prime}$ would strongly exceed the measured surface number density of all stars in case of a standard Kroupa/Chabrier IMF (Chabrier 2001; Kroupa 2001; Kroupa \& Weidner 2003). This problem does not appear in case of a top-heavy IMF. The IAC-STAR tool used to infer the top-heavy KLFs does not take pre-MS evolutionary tracks into account and therefore misses the bump of stars on the pre-MS at around $K_{\mathrm{s}}=18$. The actual surface number densities can therefore be expected to be a factor of two to three higher at this magnitude (where we roughly estimated the excess from the pre-MS onset bump in the KLF that includes pre-MS tracks). If the IMF of the 5 Myr-old stellar population is indeed as top-heavy as suggested by Bartko et al. (2010), then its contamination of our number counts can be neglected. For a less extreme IMF, as suggested by Lu et al. (2013), the contamination may reach a value up to about $40 \%$ at $R=2^{\prime \prime}$, but will rapidly diminish due to the steep decrease of the surface density of the young stars with $R$.

We have created model surface density distributions for the potential pre-MS stars, assuming two different parameters for their power-law index and for their surface density at $R=2^{\prime \prime}$. Subsequently, those models were subtracted from the star counts at $17.5 \leq K_{\mathrm{s}} \leq 18.5$ and a simple power-law was fitted to the data at 0.04 (pc) $\leq R \leq 0.5 \mathrm{pc}$. The resulting values of the projected power-law index, $\Gamma$ are listed in Table 3 . They lie in the range $\Gamma=0.12-0.22$, flatter than for the uncorrected surface density $(\Gamma=0.36 \pm 0.04$, ID 1 in Table 2$)$. We note that this

1 http://stev.oapd.inaf.it/cgi-bin/cmd 


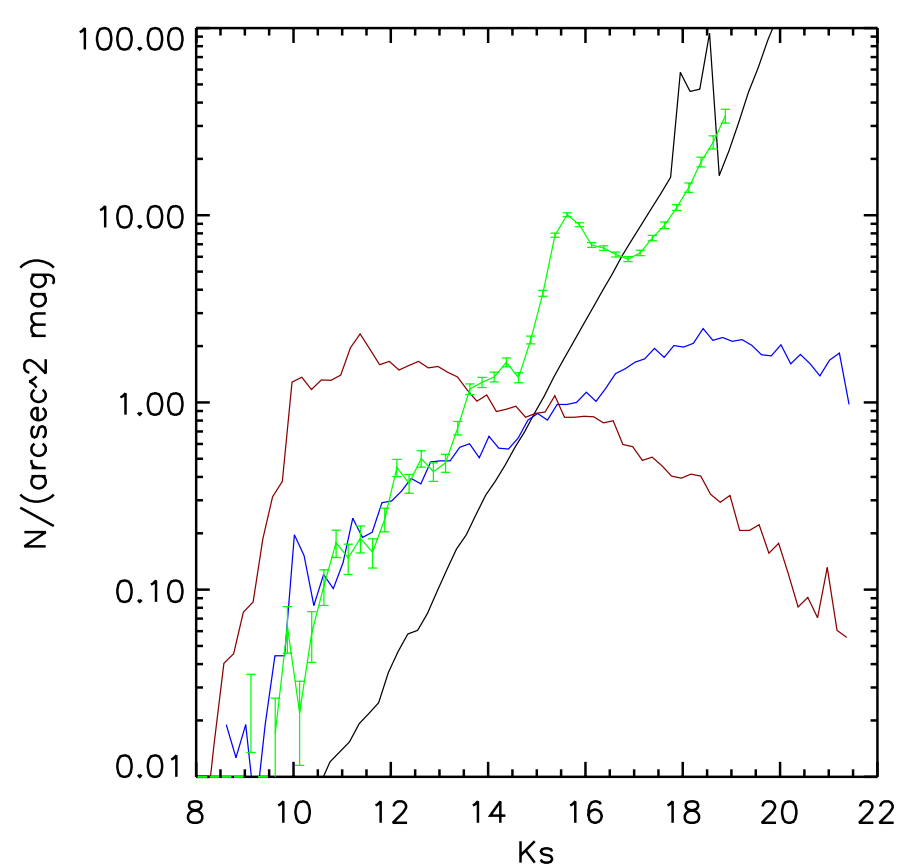

Fig. 12. Model KLFs for the youngest, $\sim 5 \mathrm{Myr}$ old stellar population at $R=2^{\prime \prime}$. Black line: Kroupa IMF; blue line: IMF from Lu et al. (2013); red line: IMF from Bartko et al. (2010). The latter two KLFs were created with a tool that does not include pre-MS evolution and therefore lack the bump at $K \approx 18$. The surface density is normalised to $R=2^{\prime \prime}$ $(0.08 \mathrm{pc})$, assuming that it rises as $R^{-1}$ towards Sgr A*. The surface density was normalised with the densities measured by Lu et al. (2013). The green line is the KLF measured from our data and normalised to the star counts at $K_{\mathrm{s}}=18$. We note that all known massive, young stars at $K_{\mathrm{s}} \lesssim 16$ are excluded from this KLF.

Table 3. Parameters used in the estimation of the surface density profile of pre-MS stars and resulting corrected $\Gamma$ for the density profile of stars with magnitudes $17.5 \leq K_{\mathrm{s}} \leq 18.5$, fitted at $0.04 \mathrm{pc} \leq R \leq 0.5 \mathrm{pc}$.

\begin{tabular}{lllll}
\hline \hline ID & $\eta^{a}$ & $\Sigma\left(2^{\prime \prime}\right)^{b}$ & $\Gamma^{c}$ & $\chi_{\text {reduced }}^{2}$ \\
\hline 1 & 0.93 & 4.0 & $0.22 \pm 0.06$ & 0.7 \\
2 & 0.93 & 6.0 & $0.13 \pm 0.07$ & 0.7 \\
3 & 1.40 & 4.0 & $0.21 \pm 0.05$ & 0.7 \\
4 & 1.40 & 6.0 & $0.12 \pm 0.06$ & 0.9 \\
\hline
\end{tabular}

Notes. We test two values of the $\eta$-parameter: $\eta=1.40$ from (Bartko et al. 2010) and $\eta=0.93$ from (Do et al. 2013), and assume the IMF of Lu et al. (2013). ${ }^{(a)}$ Power-law index of the surface-density profile for young stars. ${ }^{(b)}$ Estimated surface density of $K_{\mathrm{s}}=18$ pre-MS stars at $R=2^{\prime \prime} .{ }^{(c)}$ Power-law index of the surface-density profile of $K_{\mathrm{s}}=18$ stars after correction for pre-MS stars.

is a conservative scenario, with a high correction factor based upon the relatively steep IMF of Lu et al. (2013). When we apply the extremely top-heavy IMF of Bartko et al. (2010), we can neglect this correction for pre-MS stars. This is supported by an additional test, where we also measured the power-law index of the stars in the brightness range $16.5 \leq K_{\mathrm{s}} \leq 17.5$ at 0.04 (pc) $\leq R \leq 0.5 \mathrm{pc}$. It is $\Gamma=0.34 \pm 0.03$, consistent with the value for the fainter stars without correction for pre-MS stars (for the fitting range $0.04(\mathrm{pc}) \leq R \leq 1.0 \mathrm{pc}$ it is $\Gamma=0.41 \pm 0.02)$.

The value of $\Gamma=0.36 \pm 0.04$ that we derive for the powerlaw index of stars at $K_{\mathrm{s}} \approx 18$, using only data at a projected distance of $R \leq 0.5 \mathrm{pc}$ from Sgr A*, lies $9 \sigma$ away from a flat core. Even if we take into account the possible contamination

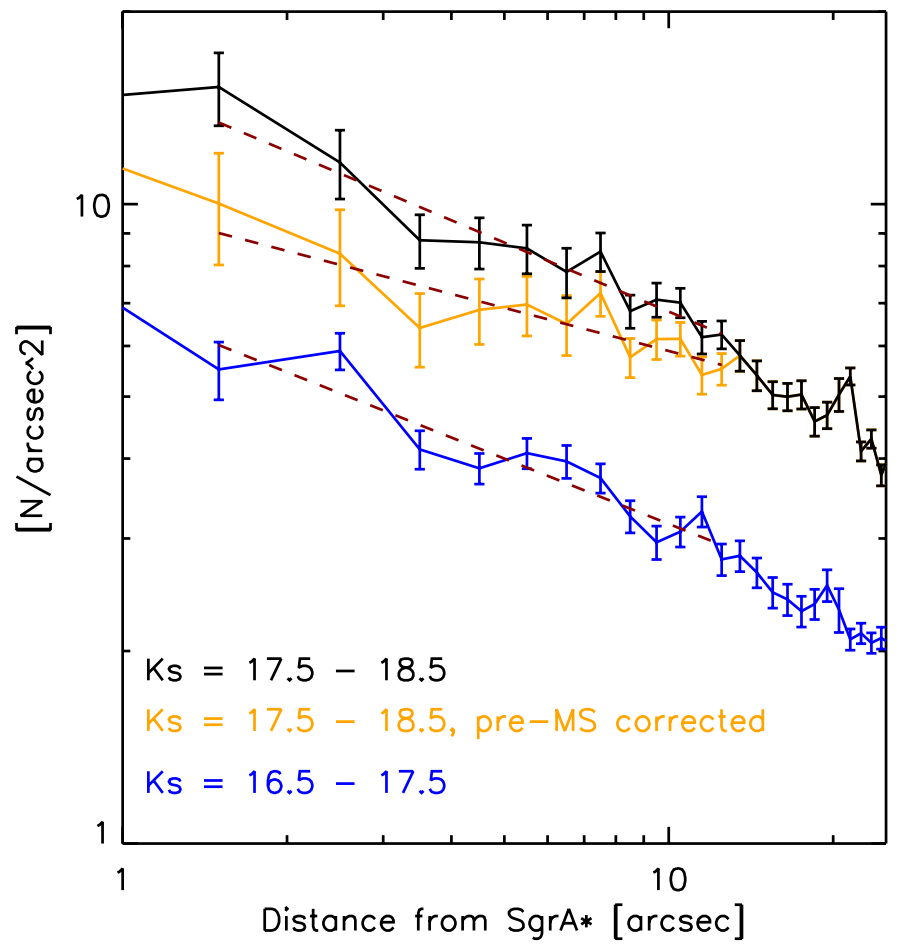

Fig. 13. Extinction and crowding-corrected surface number density profiles for $16.5 \leq K_{\mathrm{s}} \leq 17.5$ (blue line), $17.5 \leq K_{\mathrm{s}} \leq 18.5$ (black line), and for $17.5 \leq K_{\mathrm{s}} \leq 18.5$ after correction for potentially present pre-MS stars from the most recent star formation event (using parameters from ID 1 in Table 3 ). The dashed red lines are simple power-law fits in the range $0.04(\mathrm{pc}) \leq R \leq 0.5 \mathrm{pc}$. Tables with the stellar surface density data have been made available at the CDS.

by pre-MS stars, then we can still exclude a flat core, as discussed in the preceding paragraph. We therefore conclude that the contamination of the measured surface densities by pre-MS stars from the most recent star formation event is probably not significant. However, we note that the contamination by slightly older stars, from star formation about $100 \mathrm{Myr}$ ago, is probably a more important source of systematic error than the pre-MS stars, as discussed in the previous section.

\section{4. $3 D$ profile: Nuker fit}

As we could see in Sect. 4.3, the value of the projected powerlaw index depends on the radial fitting range, with a tendency to steepen at large $R$. The morphology of the cluster at large radii will impact the measured projected quantities. For example, for smaller clusters the inner projected density slope will appear flatter (see also Fig. 8 in Paper II). We know that the NSC shows a steep density decrease at $R \gtrsim 2-3$ pc (see, e.g. Launhardt et al. 2002; Schödel et al. 2014a). Also, we are dealing with a finite system, with a half-light radius on the order of 5 pc (Feldmeier et al. 2014; Schödel et al. 2014a; Fritz et al. 2016). To better constrain the shape of the NSC, we consider it therefore necessary to use a 3D model for a fit to the observed projected surface densities.

In order to convert the measured $2 \mathrm{D}$ profile into a $3 \mathrm{D}$ density law, we need to deal with projection effects, which requires us to constrain the surface density on scales larger than what we could measure with NACO. For this purpose we use the data from Fritz et al. (2016), which they acquired from observations with VLT/NACO, WFC3/HST, and VIRCAM/VISTA. 
Table 4. Best-fit model parameters for Nuker fits to faint stars.

\begin{tabular}{llllll}
\hline \hline ID & $\begin{array}{l}r_{\mathrm{b}} \\
(\mathrm{pc})\end{array}$ & $\gamma$ & $\beta$ & $\begin{array}{l}\rho\left(r_{\mathrm{b}}\right) \\
\left(\mathrm{pc}^{-3}\right)\end{array}$ & $\chi_{\text {reduced }}^{2}$ \\
\hline $1^{a}$ & $5.2 \pm 0.6$ & $1.45 \pm 0.03$ & $4.6 \pm 0.7$ & $40 \pm 7$ & 1.7 \\
$2^{b}$ & $5.0 \pm 0.5$ & $1.44 \pm 0.03$ & $4.1 \pm 0.5$ & $46 \pm 7$ & 1.7 \\
$3^{c}$ & $5.0 \pm 0.5$ & $1.44 \pm 0.03$ & $4.1 \pm 0.5$ & $46 \pm 7$ & 1.7 \\
$4^{d}$ & $4.8 \pm 0.5$ & $1.43 \pm 0.03$ & $3.5 \pm 0.3$ & $53 \pm 7$ & 1.7 \\
$5^{e}$ & $4.9 \pm 0.5$ & $1.42 \pm 0.03$ & $3.5 \pm 0.3$ & $53 \pm 7$ & 1.7 \\
$6^{f}$ & $5.0 \pm 0.7$ & $1.43 \pm 0.03$ & $3.6 \pm 0.6$ & $46 \pm 14$ & 2.0 \\
$7^{g}$ & $5.0 \pm 0.5$ & $1.46 \pm 0.03$ & $3.6 \pm 0.3$ & $46 \pm 7$ & 1.7 \\
$8^{h}$ & $5.3 \pm 0.7$ & $1.41 \pm 0.03$ & $3.7 \pm 0.4$ & $39 \pm 14$ & 1.7 \\
$9^{i}$ & $4.8 \pm 0.4$ & $1.43 \pm 0.03$ & $3.5 \pm 0.3$ & $53 \pm 7$ & 1.7 \\
$10^{j}$ & $4.3 \pm 0.5$ & $1.29 \pm 0.05$ & $3.4 \pm 0.3$ & $72 \pm 14$ & 2.0 \\
$11^{k}$ & $4.4 \pm 0.4$ & $1.29 \pm 0.05$ & $3.4 \pm 0.2$ & $72 \pm 14$ & 1.9 \\
\hline
\end{tabular}

Notes. ${ }^{(a)}$ Fit range: $0.04 \leq R \leq 20$ pc. Fore-/background emission model 1 of Table 2 in Schödel et al. (2014a). ${ }^{(b)}$ Fit range: $0.04 \leq R \leq 20$ pc. Fore-/background emission model 2 of Table 2 in Schödel et al. (2014a). ${ }^{(c)}$ Fit range: $0.04 \leq R \leq 20$ pc. Fore-/background emission model 3 of Table 2 in Schödel et al. (2014a). ${ }^{(d)}$ Fit range: $0.04 \leq R \leq 20$ pc. Fore-/background emission model 4 of Table 2 in Schödel et al. (2014a). (e) Fit range: $0.04 \leq R \leq 20$ pc. Fore-/background emission model 5 of Table 2 in Schödel et al. (2014a). ${ }^{(f)}$ Fit range: $0.04 \leq R \leq 10$ pc. Fore-/background emission model 5 of Table 2 in Schödel et al. (2014a). ${ }^{(g)}$ Fit range: $0.04 \leq R \leq 20$ pc. Fore-/background emission model 5 of Table 2 in Schödel et al. (2014a). Lower integration boundary at $r=R+0.01$ pc. ${ }^{(h)}$ Fit range: $0.04 \leq R \leq 20$ pc. Fore-/background emission model 5 of Table 2 in Schödel et al. (2014a). $\alpha=5$. $^{(i)}$ Fit range: $0.04 \leq R \leq 20$ pc. Fore-/background emission model 5 of Table 2 in Schödel et al. (2014a). $\alpha=20{ }^{(j)}$ Fit range: $0.04 \leq R \leq 20$ pc. Fore-/background emission model 5 of Table 2 in Schödel et al. (2014a). Subtracted potential contamination by pre-MS stars as in model 4 of Table $3{ }^{(k)}$ Fit range: $0.04 \leq R \leq 20$ pc. Fore-/background emission model 5 of Table 2 in Schödel et al. (2014a). Subtracted potential contamination by pre-MS stars as in model 1 of Table 3 .

To combine these data with ours, we have to assume that, on large scales, the NSC stellar population is well mixed and that its average properties (mass function) do not change. We scaled the data of Fritz et al. (2016) to ours in the range $0.5 \mathrm{pc} \leq$ $R \leq 1.0 \mathrm{pc}$. Subsequently, we subtracted an estimate of the fore-/background star density using models for the non-NSC emission from Table 2 of Schödel et al. (2014a). This simple procedure is possible because the scale lengths of the latter components are one to several orders of magnitude larger than the half-light radius of the NSC (see, e.g. Launhardt et al. 2002; Bland-Hawthorn \& Gerhard 2016).

We used a 3D Nuker model (Lauer et al. 1995) as given in Eq. (1) of Fritz et al. (2016):

$\rho(r)=\rho\left(r_{\mathrm{b}}\right) 2^{(\beta-\gamma) / \alpha}\left(\frac{r}{r_{\mathrm{b}}}\right)^{-\gamma}\left[1+\left(\frac{r}{r_{\mathrm{b}}}\right)^{\alpha}\right]^{(\gamma-\beta) / \alpha}$,

where $r$ is the $3 \mathrm{D}$ distance from $\operatorname{Sgr} \mathrm{A}^{*}, r_{\mathrm{b}}$ is the break radius, $\rho$ is the 3D density, $\gamma$ is the exponent of the inner and $\beta$ the one of the outer power-law, and $\alpha$ defines the sharpness of the transition. We fixed the parameter $\alpha=10$, but explored other values, too (e.g. $\alpha=5$ in fit ID 9 in Table 4), with the result that the precise value of $\alpha$ does not have any significant impact on the best -fit parameters, in particular on the value of $\gamma$. We projected the density onto the sky via an integral as given in Eq. (3) in Paper II and finally we fit the surface density profiles at $R \leq 20 \mathrm{pc}$. As we explain in Paper II, although Nuker model has been previously used for fitting $2 \mathrm{D}$ data, we use it as a generalisation of a broken power law in order to describe the 3D shape of the cluster. In order to determine the fore-/background star density we used the Sérsic models for the non-NSC emission listed in Table 2 of Schödel et al. (2014a) and scaled them to the data at $R \geq 20$ pc. The results of our fits are listed in Table 4 . We also performed two fits with the correction for the potential pre-MS stars (IDs 10 and 11 in Table 4). In Appendix B we explain the computation of the systematic uncertainties for the different parameters that may result from the deprojection (denoted by the

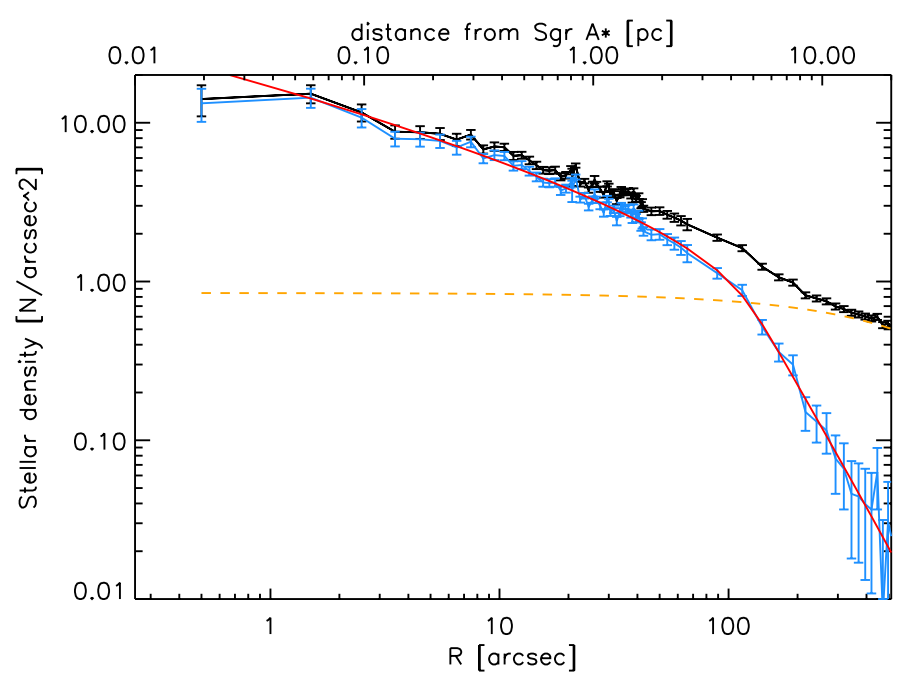

Fig. 14. Black: combined, corrected surface density data for stars in the magnitude interval $17.5 \leq K_{\mathrm{s}} \leq 18.5$ from our deep plus wide field image, complemented at large radii by scaled data from Fritz et al. (2016). The dashed orange line is a model for the emission from the nuclear disc (model 5 in Table 2 of Schödel et al. 2014a) that is subtracted from the black data points, resulting in the blue data points. The red line is a Nuker model fit (ID 5 in Table 4).

subscript sys in the following). We use the mean of the parameters and their standard deviation to obtain orientative values for the average best Nuker model: $r_{\mathrm{b}}=4.9 \pm 0.3 \pm 0.2_{\text {sys }} \mathrm{pc}$, $\gamma=1.41 \pm 0.06 \pm 0.1_{\text {sys }}, \beta=3.7 \pm 0.4 \pm 0.1_{\text {sys }}$, and a density at the break radius of $\rho\left(r_{\mathrm{b}}\right)=52 \pm 12 \mathrm{pc}^{-3}$. We note that $\rho\left(r_{\mathrm{b}}\right)$ is strongly correlated with the values of the other parameters. Its mean value is orientative and we do not cite a systematic error for this parameter. The best fit according to model ID 5 in Table 4 is shown in Fig. 14.

When we take into account the possible contamination by pre-MS stars, then there is a systematic shift towards lower 
values in the best-fit values for $r_{\mathrm{b}}, \gamma$, and $\beta$, as illustrated, for example, by fit ID 10 in Table $4: r_{\mathrm{b}}=4.3 \pm 0.5 \mathrm{pc}, \gamma=$ $1.29 \pm 0.05, \beta=3.4 \pm 0.3$.

The Nuker fit shows that the faint stars show a cusp-like distribution around $\mathrm{Sgr} \mathrm{A}^{*}$. A flat core can be excluded with high significance. We explicitly note that in the fits presented in this work we omit the region $R \leq 1^{\prime \prime}(0.04 \mathrm{pc})$. In this region, the star counts appear to drop slightly below the expected levels. However, this region is also the most crowded region, which may lead to strong systematics in the star counts. Additionally, the stellar population in the extremely close environment of Sgr A* may have been altered, as is indicated by the presence of the so called "S-stars", apparently B-type MS stars that appear concentrated within $R<1^{\prime \prime}$ of Sgr A* and may have been deposited there by individual scatter or capture events (see, e.g. Eisenhauer et al. 2005; Genzel et al. 2010; Alexander 2011).

\subsection{Distribution of giant stars near $\operatorname{Sgr} A^{*}$}

In agreement with previous work, we have found an unexpectedly flat surface density for RC stars and brighter giants within about $0.3 \mathrm{pc}$ of $\operatorname{Sgr} \mathrm{A}^{*}$. This indicates a deficit of giants in this region. Here we produce a 3D Nuker model fit for the giant stars and try to constrain the number of potentially missing giants. We proceeded as in Sect. 5.4. At large radii, we used the data from Fritz et al. (2016) as described in the preceding section. The resulting best-fit parameters are given in Table 5 .

As can be seen from the reduced $\chi^{2}$ values, the quality of the fit is significantly worse than for the faint stars, but improves as we omit the centralmost data points. We use the mean and error of the mean of the best-fit parameters in Table 5 to obtain orientative values for the average best Nuker model for giants: $r_{\mathrm{b}}=5.7 \pm 0.8 \pm 0.2_{\text {sys }} \mathrm{pc}, \gamma=1.53 \pm 0.13 \pm 0.1_{\text {sys }}$, and $\beta=3.7 \pm 0.2 \pm 0.1_{\text {sys. }}$. As we can see, the mean parameters agree within $1-2 \sigma$ with the ones determined from the Nuker fits to the faint stars, omitting fit 7 , which we believe to be not adequate (see below). The differences between the best-fit values for giants and faint stars may indicate either systematics that we have not accounted for or that the two brightness ranges do not trace populations of similar mean age and therefore dynamical state. As can be seen in Fig. 15, a projected Nuker law can provide a reasonable fit to the projected surface densities of giants down to projected distances $R \approx 0.1 \mathrm{pc}$. Also, if we consider the Nuker fits to be reasonable 0th order approximations, then they are consistent with a cusp-like 3D density distribution of the giants, in spite of the flat projected density at small $R$. Forcing a flattish inner cusp, for example by fixing $\gamma=1.0$ as in fit ID 7 in Table 5 will lead to a bad fit at large distances, as shown by the dashdotted purple line in the upper panel of Fig. 15, with parameters that deviate strongly from the best fit-parameters for all other cases (both star counts in this work and diffuse light in Paper II). We therefore argue that, in spite of the observed core projection at small $R$, the observed surface density of giants in the GC is inconsistent with such a structure.

We point out that this does not contradict previous work. Some differences can be explained by the use of different data or references to constrain the structure of the cluster on scales out to $20 \mathrm{pc}$. Also, contrary to other studies, in this work we have subtracted the projected density of stars that we do not consider to form part of the NSC proper, but to belong to the fore-and background population. We do this in order to facilitate comparison with theory, which always considers isolated systems. While our methodology may make a comparison with other publications therefore difficult, we point out that, given the
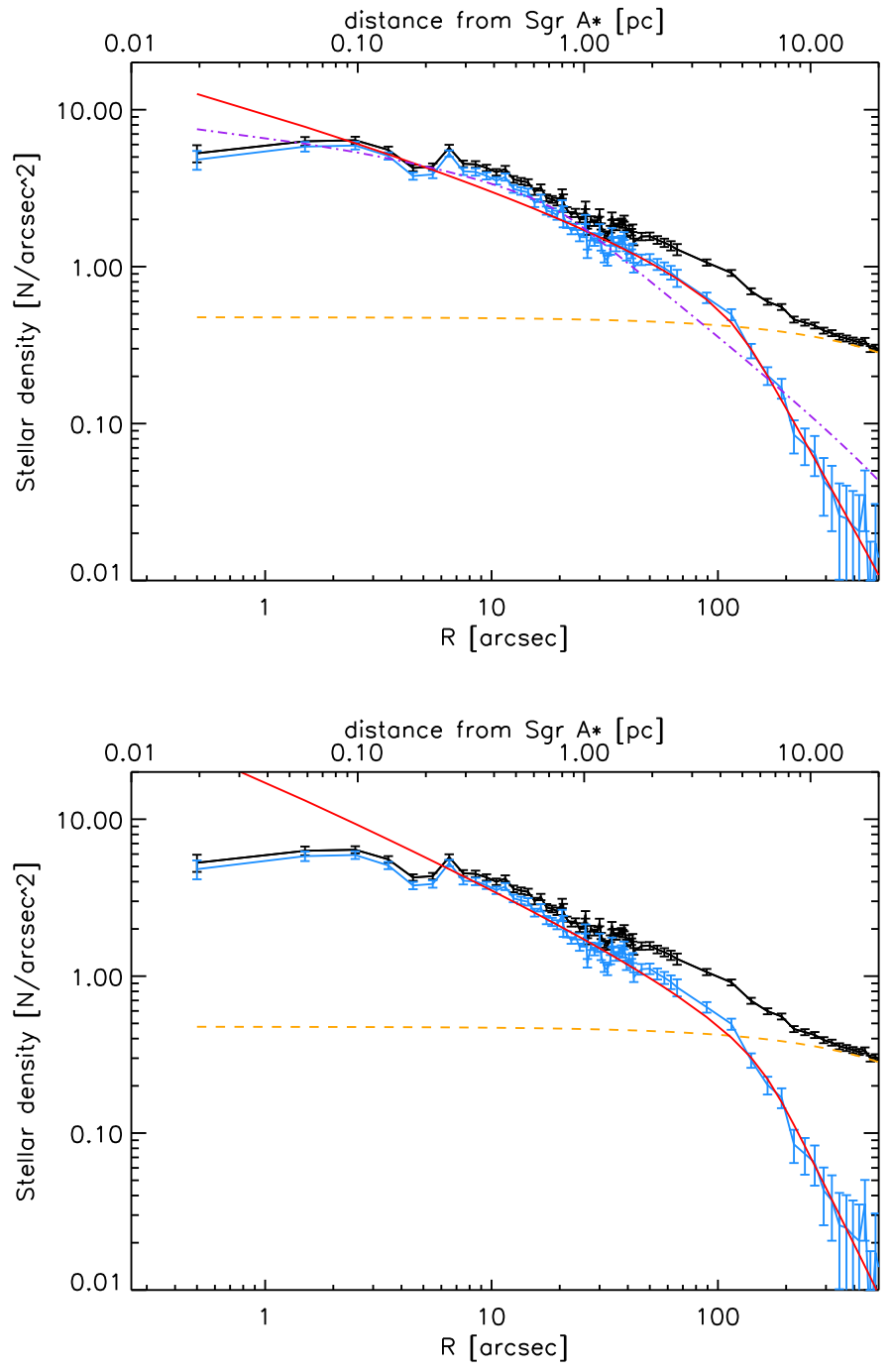

Fig. 15. Upper panel: black: combined, corrected surface density data for stars in the magnitude interval $12.5 \leq K_{\mathrm{s}} \leq 16$ from our deep plus wide field image, complemented at large radii by scaled data from Fritz et al. (2016). The orange line is a model for the emission from the nuclear disc (model 5 in Table 2 of Schödel et al. 2014a) that is subtracted from the black data points, resulting in the blue data points. The red line is a Nuker model fit (ID 1 in Table 5). The dash-dotted purple line is a Nuker model fit with $\gamma=1.0$ fixed (ID 7 in Table 5). Lower panel: like upper panel, but Nuker fit from ID 4 in Table 5.

statistical and systematic uncertainties, our $\gamma$ for the old stars still overlaps within about $2 \sigma$ with the values given by other work (e.g. Do et al. 2009; Fritz et al. 2016). Hence, while we find consistently an observed projected flat surface density of giants in the innermost few arcseconds, we find that this does not require a flat core in $3 \mathrm{D}$.

The observed flat projected profile within $\sim 0.3 \mathrm{pc}$ and the fact that the quality of the fit improves when we omit the innermost data points may indicate that something has altered the apparent distribution of giants in this region. To estimate the number of potentially "missing" giants, we focus on the region $R \leq 0.3$ pc. Since it is impossible to know what would be the "correct" number density model for giants stars, we use the following simple approach. We fit different Nuker laws to the data, where we omit the data inside $R=0.0,0.1,0.2,0.3 \mathrm{pc}$. The fit for $R=0.0$ can serve as a benchmark for the actually measured distribution, while the fits that omit data approximate the cluster 
Table 5. Best-fit model parameters for Nuker fits of old giants.

\begin{tabular}{llllll}
\hline \hline ID & $\begin{array}{l}r_{\mathrm{b}} \\
(\mathrm{pc})\end{array}$ & $\gamma$ & $\beta$ & $\begin{array}{l}\rho\left(r_{\mathrm{b}}\right) \\
\left(\mathrm{pc}^{-3}\right)\end{array}$ & $\chi_{\text {reduced }}^{2}$ \\
\hline $1^{a}$ & $5.2 \pm 0.9$ & $1.45 \pm 0.04$ & $3.6 \pm 0.5$ & $23 \pm 8$ & 5.1 \\
$2^{b}$ & $5.6 \pm 1.0$ & $1.52 \pm 0.04$ & $3.7 \pm 0.6$ & $19 \pm 7$ & 4.7 \\
$3^{c}$ & $6.3 \pm 1.2$ & $1.63 \pm 0.04$ & $3.8 \pm 0.7$ & $14 \pm 5$ & 3.8 \\
$4^{d}$ & $6.8 \pm 1.3$ & $1.70 \pm 0.05$ & $3.9 \pm 0.7$ & $11 \pm 5$ & 3.3 \\
$5^{e}$ & $5.8 \pm 1.0$ & $1.50 \pm 0.00$ & $3.7 \pm 0.6$ & $18 \pm 6$ & 5.2 \\
$6^{f}$ & $4.5 \pm 0.9$ & $1.37 \pm 0.04$ & $3.4 \pm 0.5$ & $32 \pm 11$ & 6.4 \\
$7^{g}$ & $0.9 \pm 0.1$ & $1.00 \pm 0.00$ & $2.2 \pm 0.1$ & $572 \pm 89$ & 7.2 \\
\hline
\end{tabular}

Notes. ${ }^{(a)}$ Fit range: $0.04 \leq R \leq 20$ pc. Fore-/background emission model 5 of Table 2 in Schödel et al. (2014a). ${ }^{(b)}$ Fit range: $0.1 \leq R \leq 20$ pc. Fore-/background emission model 5 of Table 2 in Schödel et al. (2014a). ${ }^{(c)}$ Fit range: $0.2 \leq R \leq 20$ pc. Fore-/background emission model 5 of Table 2 in Schödel et al. (2014a). ${ }^{(d)}$ Fit range: $0.3 \leq R \leq 20$ pc. Fore-/background emission model 5 of Table 2 in Schödel et al. (2014a). ${ }^{(e)}$ Fit range: $0.04 \leq R \leq 20$ pc. $\gamma=1.5$ fixed. Fore-/background emission model 5 of Table 2 in Schödel et al. $(2014 \mathrm{a})$. ${ }^{(f)}$ Fit range: $0 \leq R \leq 20$ pc. Fore-/background emission model 5 of Table 2 in Schödel et al. (2014a). ${ }^{(g)}$ Fit range: $0.0 \leq R \leq 20$ pc. $\gamma=1.0$ fixed. Fore-/background emission model 5 of Table 2 in Schödel et al. (2014a).

structure without potentially missing stars at small $R$. The benchmark fit is shown in the upper panel of Fig. 15, and the fit omitting data at $R \leq 0.3 \mathrm{pc}$ is shown in the lower panel of the same figure.

Subsequently, we compute the amount of stars at $r<0.2 \mathrm{pc}$ for each model and compare it to the benchmark solution. The difference in number provides the estimate of possibly missing stars at $r<0.2 \mathrm{pc}$. For the three fits that omit data at small R, this numbers varies between 40 to 200 . While the uncertainty of this crude estimate is high, it provides us with an idea of the order of magnitude of the problem. The lesson to take away here is, in our opinion, that any mechanisms that intends to explain the deficit of giant stars near Sgr A* should be able to be efficient enough to account for roughly 100 missing giants.

\subsection{Comparison to other work and discussion}

Consistent with previous work, we find indications of a flattening of the density profile of the RC stars and brighter giants inside $R \approx 8^{\prime \prime} / 0.3$ pc (Buchholz et al. 2009; Bartko et al. 2010; Do et al. 2013). Also, our results agree well with the fits shown for RC stars in Schödel et al. (2007, their Fig. 17). We also note that the density profile of stars about a magnitude fainter than the RC was analysed in the latter work. Although Schödel et al. (2007) used a broken power-law, it is clear that a single power-law, with $\Gamma \approx 0.4$, would also provide a satisfactory fit to the stars fainter than the RC (see right panel in their Fig. 17). Yusef-Zadeh et al. (2012) determined the surface light density of faint stars in HST/NICMOS images at 1.45, 1.70, and $1.90 \mu \mathrm{m}$. After the masking of bright sources, the stars that dominate the light profiles presented in Yusef-Zadeh et al. (2012) are probably slightly fainter than the RC and show a single powerlaw profile with $\Gamma=0.34 \pm 0.04$ at $5^{\prime \prime} \leq R \leq 0.7^{\prime \prime}$, in agreement with what we find in this work and with the surface density of stars fainter than the RC shown in Schödel et al. (2007). To conclude, our analysis agrees well with previously published results.

There are also several key differences between previous work and our analysis, which goes beyond the existing state-of-the-art:

1. We reach about one magnitude deeper in the KLF and are not limited/dominated by stars in the RC or bright giants.

2. We focus explicitly on well defined brightness ranges to have a constraint on the ages and masses of the stars that we are studying, while most previous work generally reported surface density profiles for broad ranges of luminosities (but was mostly dominated by RC stars).

3. We include high angular resolution data on larger radii, out to $R \approx 2$ pc (the only other work to do so was by Fritz et al. 2016).

4. We include the most recent data on the large-scale structure of the NSC and thus take into account its global structure. Much previous work was focussed on the innermost region near Sgr A*, e.g. $R<20^{\prime \prime}$ (Buchholz et al. 2009), $R \leq 5^{\prime \prime}$ (Yusef-Zadeh et al. 2012), or $R \leq 4^{\prime \prime}$ (Do et al. 2009). While these studies found inconsistencies with the existence of a stellar cusp around Sgr A* (with the exception of Yusef-Zadeh et al. 2012), they not only were dominated by bright stars (see above), but also did not take the global picture into account. A stellar cusp is expected to be well developed inside the radius of influence, $r_{\text {infl }}$, of a massive black hole, which is on the order $r_{\text {infl }}=3 \mathrm{pc}$ for Sgr A* (Alexander 2005; Merritt 2010; Feldmeier et al. 2014; Chatzopoulos et al. 2015a; Feldmeier-Krause et al. 2017).

The features of the present work have allowed us to infer a more complete picture of the stellar distribution around Sgr A*. In particular, we could show that the surface density distribution of stars fainter than the RC are inconsistent with a flat, core-like distribution with high significance, even if we take into account the possible contamination of the faint star counts by pre-MS stars from the last star formation event. An important caveat is, however, that the faint stars analysed here may be contaminated considerably by dynamically young stars from star formation $\sim 100$ Myr ago. Unfortunately, we cannot quantitatively estimate the effect of this contamination. As concerns the old, giant stars, we find that in spite of their flat projected density at $R \leq 0.3 \mathrm{pc}$ their $3 \mathrm{D}$ distribution is consistent with a stellar cusp and definitely inconsistent with a flat core.

In Paper II we study the diffuse stellar light density - probably arising from stars in the magnitude range $K_{\mathrm{s}}=19-20$. We find that the projected diffuse light density can be described well with a simple power-law with an index $\Gamma_{\text {diffuse }}=0.28 \pm 0.03$, roughly in agreement with, albeit flatter than, the value of $\Gamma$ found for the surface number density of faint stars in this work. The differences between the surface density profiles of the stars in these different magnitude ranges can indicate a different age composition (see Fig. 11). The differences between the values of $\Gamma$ may also give us an idea of the real uncertainty of our 
measurements $\Gamma$, given that both methods may be biased by unknown systematic biases. We thus estimate that a realistic $1 \sigma$ uncertainty of $\Gamma$ is on the order $\sim 0.1$. For the Nuker fits of the 3D density, we also find roughly similar values between the $K_{\mathrm{s}} \approx 18$ stellar population and the fainter, unresolved one studied in Paper II. For the former, we find an inner 3D power-law index of $\gamma_{\text {resolved }}=1.41 \pm 0.06 \pm 0.1_{\text {sys }}$, for the latter we find $\gamma_{\text {unresolved }}=1.13 \pm 0.02 \pm 0.05$ sys. This allows us to claim that there exists a stellar cusp around the massive black hole Sgr A*. A flat core can be rejected with very high confidence.

The values for the break radius inferred from faint star counts and from diffuse light in Paper II are different $r_{\mathrm{b} \text {,resolved }}=5.0 \pm$ $0.2 \pm 0.2_{\text {sys }}$ and $r_{\text {b,unresolved }}=4.3 \pm 0.5$, but agree within their uncertainties. We note that the values for $r_{\mathrm{b}}$ derived here are significantly different from what was estimated by Fritz et al. (2016). This is because the surface density in the latter work was dominated by giants/RC stars in the innermost regions. Also, Fritz et al. (2016) fit the surface density of all stars in the GC field (including those from the Galactic bar and nuclear disc), while we subtracted a model to only take stars in the NSC into account. We point out that the Nuker laws presented here are optimised to describe the intrinsic properties of the nuclear cluster, but not the overall stellar density at the GC. The NSC lies, of course, embedded in the overall surrounding structures (e.g. nuclear disc, Galactic bar and Galactic bulge). The work of Fritz et al. (2016) or Schödel et al. (2014a) provides data that include the large scale stellar structures.

\section{Conclusions}

In this work we have revisited the question of the distribution of stars around the massive black hole at the centre of the Milky Way because previous studies had come up with the unexpected result that there did perhaps not exist any stellar cusp. To overcome existing limitations, we used improved analysis techniques and deep, stacked images. We could push the completeness limit of star counts about one magnitude deeper than what had been done before, to $K_{\mathrm{s}} \approx 18$. This has allowed us to study a stellar population that consists probably primarily of several Gyr old, $1-2 M_{\odot}$ stars.

Contrary to the flattening of the density distribution of giant stars near Sgr A*, a well known observation that we also reproduced here, the projected surface density profile of the $K_{\mathrm{s}} \approx 18$ and $K_{\mathrm{s}} \approx 17$ stars can be described very well by a single powerlaw. We estimate a power-law index of $\Gamma=0.47 \pm 0.07$. This value may be up to $\sim 0.3$ lower if the star counts are heavily contaminated by pre-MS stars from the most recent star formation event. Given the probably highly top-heavy IMF of this most recent star formation event, for which we present some additional evidence in this paper (Fig. 12), a strong contamination seems unlikely, however.

Both the faint resolved stars and the even fainter, unresolved stars studied in Paper II show consistently projected power-law surface densities around Sgr A*. We can thus exclude a corelike stellar density distribution with high confidence. Unfortunately, our current best knowledge of the star formation history within $1 \mathrm{pc}$ of $\mathrm{Sgr} \mathrm{A}^{*}$ implies that a large fraction of the faint stars may be dynamically unrelaxed. Therefore we cannot claim unambiguously the existence of a stellar cusp of old, dynamically relaxed stars around Sgr A*. However, the fact that we find power-law cusps for faint stars in two different brightness bins as well as for the diffuse emission that arises from low-mass, probably old and dynamically relaxed stars (Paper II), makes the existence of a relaxed cusp highly plausible in our opinion.
We fit 3D Nuker models to the data to estimate the intrinsic structure of the Milky Way's NSC. The break radius of the Nuker model lies at about $5 \mathrm{pc}$, somewhat larger than the radius of influence of Sgr A*. This agrees with theoretical expectations that the cusp is visible well inside the break radius (e.g. Alexander 2005).

The stellar cusp around Sgr A* inferred from the star counts may be somewhat flatter than the theoretically expected value of $\gamma_{\text {theor }}=1.5$ for the low-mass stellar component in a multi-mass cluster (see Alexander 2005, and references therein). Some disagreement with theory is to be expected, however. After all, the Milky Way's NSC is more complex than what was considered in existing theoretical work. For example, it is embedded in a complex environment, has a complex star formation history, and may have suffered events of infall and accretion of smaller clusters (e.g. Pfuhl et al. 2011; Feldmeier et al. 2014; Schödel et al. 2014 b). New $N$-body simulations, that were undertaken in parallel to this observational work (Paper III) and that included the effect of repeated star formation events, find flat cusp slopes in agreement with our findings.

Finally, the existence of a stellar cusp implies that the giant stars around Sgr A* do indeed display a deficiency in numbers within a projected radius of a few $0.1 \mathrm{pc}$. We estimate that on the order 100 giants may be "missing". This region overlaps with the region where we find young massive stars that may have formed in a dense gas disc a few Myr ago. Repeated collisions with proto-stellar clumps in this disc may have stripped the giants of their envelopes, rendering them thus unobservable (Amaro-Seoane \& Chen 2014; Kieffer \& Bogdanović 2016).

Acknowledgements. The research leading to these results has received funding from the European Research Council under the European Union's Seventh Framework Programme (FP7/2007-2013)/ERC grant agreement No. [614922]. This work is based on observations made with ESO Telescopes at the La Silla Paranal Observatory under programmes IDs 183.B-0100 and 089.B-0162. We thank T. Fritz for detailed and valuable comments. This work has made use of the IAC-STAR Synthetic CMD computation code. IAC-STAR is suported and maintained by the computer division of the Instituto de Astrofísica de Canarias. PAS acknowledges support from the Ramón y Cajal Programme of the Ministry of Economy, Industry and Competitiveness of Spain. This work has been partially supported by the CAS President's International Fellowship Initiative.

\section{References}

Aharon, D., \& Perets, H. B. 2015, ApJ, 799, 185

Alexander, T. 2005, Phys. Rep., 419, 65

Alexander, T. 2011, in The Galactic Center: a Window to the Nuclear Environment of Disk Galaxies, eds. M. R. Morris, Q. D. Wang, \& F. Yuan, ASP Conf. Ser., 439, 129

Alexander, T., \& Hopman, C. 2009, ApJ, 697, 1861

Amaro-Seoane, P., \& Chen, X. 2014, ApJ, 781, L18

Amaro-Seoane, P., Freitag, M., \& Spurzem, R. 2004, MNRAS, 352, 655

Aparicio, A., \& Gallart, C. 2004, AJ, 128, 1465

Bahcall, J. N., \& Wolf, R. A. 1976, ApJ, 209, 214

Bartko, H., Martins, F., Trippe, S., et al. 2010, ApJ, 708, 834

Baumgardt, H., Makino, J., \& Ebisuzaki, T. 2004, ApJ, 613, 1143

Baumgardt, H., Amaro-Seoane, P., \& Schoedel R. 2018, A\&A, 609, A28 (Paper III)

Bessell, M. S. 1990, PASP, 102, 1181

Bland-Hawthorn, J., \& Gerhard, O. 2016, ARA\&A, 54, 529

Blum, R. D., Ramírez, S. V., Sellgren, K., \& Olsen, K. 2003, ApJ, 597, 323

Boehle, A., Ghez, A. M., Schödel, R., et al. 2016, ApJ, 830, 17

Böker, T. 2010, in IAU Symp. 266, eds. R. de Grijs, \& J. R. D. Lépine, 58

Bressan, A., Marigo, P., Girardi, L., et al. 2012, MNRAS, 427, 127

Buchholz, R. M., Schödel, R., \& Eckart, A. 2009, A\&A, 499, 483

Chabrier, G. 2001, ApJ, 554, 1274

Chatzopoulos, S., Fritz, T. K., Gerhard, O., et al. 2015a, MNRAS, 447, 948 Chatzopoulos, S., Gerhard, O., Fritz, T. K., et al. 2015b, MNRAS, 453, 939 
Chen, Y., Girardi, L., Bressan, A., et al. 2014, MNRAS, 444, 2525 Dale, J. E., Davies, M. B., Church, R. P., \& Freitag, M. 2009, MNRAS, 393, 1016

Diolaiti, E., Bendinelli, O., Bonaccini, D., et al. 2000, A\&AS, 147, 335

Do, T., Ghez, A. M., Morris, M. R., et al. 2009, ApJ, 703, 1323

Do, T., Lu, J. R., Ghez, A. M., et al. 2013, ApJ, 764, 154

Do, T., Kerzendorf, W., Winsor, N., et al. 2015, ApJ, 809, 143

Eisenhauer, F., Genzel, R., Alexander, T., et al. 2005, ApJ, 628, 246

Feldmeier, A., Neumayer, N., Seth, A., et al. 2014, A\&A, 570, A2

Feldmeier-Krause, A., Neumayer, N., Schödel, R., et al. 2015, A\&A, 584, A2

Feldmeier-Krause, A., Zhu, L., Neumayer, N., et al. 2017, MNRAS, 466, 4040

Fritz, T. K., Gillessen, S., Dodds-Eden, K., et al. 2011, ApJ, 737, 73

Fritz, T. K., Chatzopoulos, S., Gerhard, O., et al. 2016, ApJ, 821, 44

Genzel, R., Schödel, R., Ott, T., et al. 2003, ApJ, 594, 812

Genzel, R., Eisenhauer, F., \& Gillessen, S. 2010, Rev. Mod. Phys., 82, 3121

Gillessen, S., Plewa, P., Eisenhauer, F., et al. 2017, ApJ, 837, 30

Gültekin, K., Richstone, D. O., Gebhardt, K., et al. 2009, ApJ, 698, 198

Kieffer, T. F., \& Bogdanović, T. 2016, ApJ, 823, 155

Knuth, K. H. 2006, ArXiv e-prints [arXiv:physics/0605197]

Kroupa, P. 2001, MNRAS, 322, 231

Kroupa, P., \& Weidner, C. 2003, ApJ, 598, 1076

Lauer, T. R., Ajhar, E. A., Byun, Y.-I., et al. 1995, AJ, 110, 2622

Launhardt, R., Zylka, R., \& Mezger, P. G. 2002, A\&A, 384, 112

Lightman, A. P., \& Shapiro, S. L. 1977, ApJ, 211, 244

Löckmann, U., Baumgardt, H., \& Kroupa, P. 2010, MNRAS, 402, 519
Lu, J. R., Do, T., Ghez, A. M., et al. 2013, ApJ, 764, 155

Maíz Apellániz, J. 2006, AJ, 131, 1184

Merritt, D. 2010, ApJ, 718, 739

Meyer, L., Ghez, A. M., Schödel, R., et al. 2012, Science, 338, 84

Murphy, B. W., Cohn, H. N., \& Durisen, R. H. 1991, ApJ, 370, 60

Nishiyama, S., Tamura, M., Hatano, H., et al. 2009, ApJ, 696, 1407

Nishiyama, S., Schödel, R., Yoshikawa, T., et al. 2016, A\&A, 588, A49

Paumard, T., Genzel, R., Martins, F., et al. 2006, ApJ, 643, 1011

Pfuhl, O., Fritz, T. K., Zilka, M., et al. 2011, ApJ, 741, 108

Preto, M., \& Amaro-Seoane, P. 2010, ApJ, 708, L42

Schödel, R. 2010, A\&A, 509, A58

Schödel, R., Eckart, A., Alexander, T., et al. 2007, A\&A, 469, 125

Schödel, R., Merritt, D., \& Eckart, A. 2009, A\&A, 502, 91

Schödel, R., Najarro, F., Muzic, K., \& Eckart, A. 2010, A\&A, 511, A18

Schödel, R., Yelda, S., Ghez, A., et al. 2013, MNRAS, 429, 1367

Schödel, R., Feldmeier, A., Kunneriath, D., et al. 2014a, A\&A, 566, A47

Schödel, R., Feldmeier, A., Neumayer, N., Meyer, L., \& Yelda, S. 2014b, Class. Quant. Grav., 31, 244007

Schödel, R., Gallego-Cano, E., Dong, H., et al. 2018, A\&A, 609, A27 (Paper II)

Støstad, M., Do, T., Murray, N., et al. 2015, ApJ, 808, 106

Tang, J., Bressan, A., Rosenfield, P., et al. 2014, MNRAS, 445, 4287

Trippe, S., Gillessen, S., Gerhard, O. E., et al. 2008, A\&A, 492, 419

Witzel, G., Eckart, A., Bremer, M., et al. 2012, ApJS, 203, 18

Yusef-Zadeh, F., Bushouse, H., \& Wardle, M. 2012, ApJ, 744, 24 
E. Gallego-Cano et al.: The distribution of stars around the Milky Way's central black hole. I.

\section{Appendix A: Systematic errors of the 2D fit of the Surface Density Profile}

In this appendix we examine some of the potential sources of systematic errors in the computation of the power law indices for the surface density fits.

\section{A.1. Starfinder parameters, extinction, and completeness}

There can be no absolute certainty in the reliability of source detection. For that reason, we analysed the images with different values of the StarFinder parameters, as described in Sect. 2.4. All error bars used in this work include the uncertainties due to different choices of StarFinder parameters, as well as the uncertainties of extinction and completeness corrections.

\section{A.2. Binning}

We analyse the results considering different ways of binning the data. As we see in Sect. 4.1, we study the maximum for the RLP for the star number and we obtain the best bin size for our sample. We also test other values of binning $\left(0.5^{\prime \prime}, 1^{\prime \prime}, 1.5^{\prime \prime}\right)$ and we obtain a value of the systematic error in the index of the power law less than 0.01 for RC stars and less than 0.001 for fainter stars(17.5 $\left.\leq K_{\mathrm{s}} \leq 18.5\right)$. We conclude that binning is not any significant source of systematic errors in our analysis.

\section{A.3. Fitting range}

As we can see in Table 2, the assumed fitting range can lead to significant variations in the best value of the projected power-law index $\Gamma$. It is on the order $\Delta \Gamma=0.1$.

\section{A.4. Correction for young stars}

When we study the distribution of fainter stars we have to consider the possibility that our stellar surface number density is contaminated by pre-MS stars from the latest star formation event, as discussed in Sect.5.3. As we see, we model the surface density profile of the young stars by a simple power-law and compute the number of young stars considering different scenarios. The uncertainty of this correction depends primarily on the assumed surface density distribution and mass of the young stars and is on the order of 0.05 . While the precise age of the cluster does not matter, the assumed IMF is paramount. Here, we assumed the IMF of Lu et al. (2013) as a conservative case. If the IMF is chosen as top-heavy as in Bartko et al. (2010), then the contamination by pre-MS stars can be considered insignificant. 


\section{Appendix B: Systematic errors of the 3D fit of the Surface Density Profile}

In this section we examine several potential sources of systematic errors in the computation of the power law indices for the fits of the 3D density (see Sect. 5.4) for the two analysed ranges of old stars: RC and fainter stars.

\section{B.1. Subtracted contribution from the nuclear disc}

When fitting the Nuker profiles we assume different models for the contribution of stars that do not belong to the nuclear cluster. Table 4 lists the best-fitting parameters under those different assumptions, which are included in our final error estimation.

\section{B.2. Correction for young stars}

When we compare models 5 and 11 in Table 4 we can see that the contamination by pre-MS stars can change the best-fit value of $\gamma$ by about 0.1 dex.

\section{B.3. Uncertainties from deprojection}

As we see in Sect. 5.4, we are interested in studying the 3D structure of the old cluster. We need to convert the measured 2D profile into a 3D density, so we need to consider the source of uncertainty from deprojection. For this purpose, a 3D cluster was simulated in order to project it and apply our procedure for density estimation. We proceed as follows:

1. Different 3D clusters are simulated, where 1000000 stars are distributed following a 3D Nuker model (Eq. (1)). In order to explore which parameters in the Nuker fit are more sensitive to the variations of the model, we test different cluster: clusters with a systematic variation in the break radius $\left(r_{\mathrm{b}}=1.6,5.8,6.4\right)$, clusters with a systematic variation in the exponent of the inner power-law $(\gamma=0.68,1.74,2.32)$ and clusters with a systematic variation in the exponent of the outer power-law $(\beta=4.8,6.4)$.

2. Extraction of 100 randomised samples from each model with the same star number of our sample.

3. Computation of projected density.

4. Apply the fit to the samples.

5. Comparison the input parameters in the simulation and computed parameters in the samples for each of the different models.

Table B. 1 shows the results. We can see that $\gamma$ is the least sensitive parameter to the break radius variations or to beta exponent variations of the model $(\sigma(\gamma)<0.1)$. Only in the case of $\gamma=0.58$ we can see a large difference between the gamma input parameter and the recovered ones. In general, the break radius parameter is the most sensitive to variations of the model. As we can see, if we ignore the model with $\gamma=0.58$ as an outlier (it is basically excluded by our data), safe assumptions for the systematic uncertainties due to deprojection are $\Delta \gamma \approx 0.1$; for $\Delta \beta \approx 0.1$, and $\Delta r_{\mathrm{b}} \approx 0.2$.

Table B.1. 3D cluster simulations to analyse the effect of the deprojection from 3D cluster into a $2 \mathrm{D}$ one.

\begin{tabular}{|c|c|c|c|c|c|c|c|c|c|}
\hline ID & $r_{\mathrm{b}}$ & $\gamma$ & $\beta$ & $\sigma\left(r_{\mathrm{b}}-r_{\mathrm{b} 0}\right)$ & $\operatorname{median}\left(r_{\mathrm{b}}-r_{b 0}\right)$ & $\sigma\left(\gamma-\gamma_{0}\right)$ & $\operatorname{median}\left(\gamma-\gamma_{0}\right)$ & $\sigma\left(\beta-\beta_{0}\right)$ & $\operatorname{median}\left(\beta-\beta_{0}\right)$ \\
\hline 1 & 3.20 & 1.16 & 3.20 & 0.15 & -0.01 & 0.09 & -0.05 & 0.07 & 0.01 \\
\hline 2 & 1.60 & 1.16 & 3.20 & 0.06 & 0.02 & 0.08 & -0.01 & 0.03 & 0.05 \\
\hline 3 & 4.80 & 1.16 & 3.20 & 0.32 & 0.04 & 0.09 & -0.05 & 0.14 & -0.05 \\
\hline 4 & 6.40 & 1.16 & 3.20 & 0.67 & -0.23 & 0.12 & -0.11 & 0.36 & -0.18 \\
\hline 5 & 3.20 & 0.58 & 3.20 & 0.18 & -0.04 & 0.19 & -0.21 & 0.07 & 0.02 \\
\hline 6 & 3.20 & 1.74 & 3.20 & 0.19 & 0.12 & 0.04 & 0.09 & 0.08 & 0.03 \\
\hline 7 & 3.20 & 2.32 & 3.20 & 0.37 & 0.81 & 0.02 & 0.18 & 0.10 & 0.09 \\
\hline 8 & 3.20 & 1.16 & 4.80 & 0.06 & 0.13 & 0.04 & 0.03 & 0.07 & 0.25 \\
\hline 9 & 3.20 & 1.16 & 6.40 & 0.04 & 0.11 & 0.03 & 0.01 & 0.11 & 0.34 \\
\hline
\end{tabular}

Notes. The three first columns show the values of the Nuker parameters adopted for the simulations. The last six columns show the standard deviation and the median of the parameters obtained with our fit for each of the samples of each of the different model clusters. 